\title{
GENERATION OF MAGNETIC FIELDS BY THERMOMAGNETIC EFFECTS IN A NONUNIFORMLY ROTATING LAYER OF AN ELECTRICALLY CONDUCTIVE FLUID
}

\author{
M. I. Kopp ${ }^{1}$ (D), K. N. Kulik ${ }^{1}$, A. V. Tur ${ }^{3}$ (D) V. V. Yanovsky'1, (D) \\ ${ }^{1}$ Institute for Single Crystals, NAS Ukraine, 60, Nauky Ave., Kharkiv, UA-61001, Ukraine, \\ ${ }^{2}$ V. N. Karazin Kharkiv National University, 4, Svobody Sq., Kharkiv, UA-61022, Ukraine, \\ ${ }^{3}$ Université de Toulouse [UPS], CNRS, Institut de Recherche en Astrophysique et Planétologie, \\ 9 avenue du Colonel Roche, BP 44346, 31028 Toulouse Cedex 4, France
}

(Received 26 February 2021; accepted 03 April 2021; published online 03 June 2021)

\begin{abstract}
This paper studies, the generation of magnetic fields in a nonuniformly rotating layer of finite thickness of an electrically conducting fluid by thermomagnetic (TM) instability. This instability arises due to the temperature gradient $\nabla T_{0}$ and thermoelectromotive coefficient gradient $\nabla \alpha$. The influence of the generation of a toroidal magnetic field by TM instability on the convective instability in a nonuniformly rotating layer of an electrically conductive fluid in the presence of a vertical constant magnetic field $\mathbf{B}_{0} \| \mathrm{OZ}$ is established. By applying the method of perturbation theory for the small parameter $\epsilon=\sqrt{\left(\mathrm{Ra}-\mathrm{Ra}_{c}\right) / \mathrm{Ra}_{c}}$ of the supercriticality of the stationary Rayleigh number $\mathrm{Ra}_{c}$, a nonlinear equation of the Ginzburg-Landau type was obtained. This equation describes the evolution of the finite amplitude of perturbations. Numerical solutions of this equation made it possible to determine the heat transfer in the fluid layer with and without TM effects. It is shown that the amplitude of the stationary toroidal magnetic field noticeably increases with allowance for TM effects.
\end{abstract}

Key words: thermoelectromotive force, generation of magnetic fields, Rayleigh-Benard convection, weakly nonlinear theory, Ginzburg-Landau equation.

DOI: https://doi.org/10.30970/jps.25.2401

\section{INTRODUCTION}

Magnetic fields in the Universe are observed an various cosmological scales from small planets to galaxies. The question of the origin of magnetic fields in various astrophysical objects is dealt with by the dynamo theory, which was developed as an independent section of magnetohydrodynamics. Initailly, the term "dynamo" was coined by Sir Larmor [1]. In his opinion, the hydrodynamic motion of an electroconductive fluid can generate a magnetic field by acting as a dynamo. In the modern sense, the dynamo mechanism represents the generation of a large-scale magnetic field due to the combined action of the differential (nonuniform) rotation of an astrophysical object and the mirror asymmetry of turbulent or convective flows. The topological characteristic of such flows is helicity $J_{s}=\overline{\mathbf{v} \operatorname{rot} \mathbf{v}}$, which is a measure of the knottedness of vortex field force lines [2]. The generation of the mean field occurs under the action of a turbulent e.m.f. proportional to the mean magnetic field $\mathcal{E}=\alpha_{\mathrm{h}} \overline{\mathbf{H}}$. The coefficient $\alpha_{\mathrm{h}}$ is proportional to the average helicity of the velocity field $\alpha_{\mathrm{h}} \sim \overline{\mathbf{v} \operatorname{rot} \mathbf{v}}$ and it is called the $\alpha$-effect in the literature [3]. The large-scale generation is usually called $\alpha_{\mathrm{h}}^{2}$-dynamo in the absence of differential rotation, and it is called $\alpha_{\mathrm{h}} \Omega$-dynamo in the presence of the rotation. The most developed at the moment is the dynamo theory in the kinematic formulation $[2-10]$.

Of no less importance are issues related to the selfexcitation of magnetic fields by external e.m.f. As shown by Schlüter and Biermann [11], the inhomogeneity of the chemical composition of a space object can lead to non-parallelism of the gradients of the electron pressure $\nabla p_{e}$ and electron concentration $\nabla n_{e}$. The result is a "battery" electric field $\mathbf{E}^{(i)}=\left(1 / e n_{e}\right) \nabla p_{e}$, which leads to the excitation of magnetic fields $\partial \mathbf{B} / \partial t \approx \operatorname{rot} \mathbf{E}^{(i)}$. Such a mechanism is associated with the generation of initial magnetic fields, which at some moment were completely absent. In this case, it is not always rightly assumed that only weak initial fields arise [4], which are necessary to turn on the dynamo. Numerical estimates carried out in [12] showed that the magnetic fields of the Earth and planets are created by thermoelectric currents that flow in a highly conductive region inside the planet. The temperature gradients $\nabla T$ and the gradient of the thermoelectromotive force coefficient $\nabla \alpha$ must be directed at an angle to each other. Then, the magnetic field is excited $\partial \mathbf{B} / \partial t \approx[\nabla T \times \nabla \alpha]$ by analogy with the "battery" effect of Biermann-Schlüter because of the non-parallelism of the vectors $\nabla T$ and $\nabla \alpha$. In the work [12] it is noted that Braginsky's conclusion [17] about the insignificance of thermopower in the Earth's core is unfounded. In [13] it was assumed that the temperature gradient and the gradient of the thermoelectromotive force coefficient are parallel $\left[\nabla T_{0} \times \nabla \alpha\right]=0$. However, as shown in this paper, under the condition $\left[\nabla T_{0} \times \nabla \alpha\right]=0$ the generation of magnetic fields is also possible due to the development of thermomagnetic instability (TMI). Thus, there are no contradictions with the Braginsky dynamo in this case.

Astrophysical applications of TMI are discussed in detail in review [14], where an explanation of the appearance of strong magnetic fields in the cores of white dwarfs, binary systems, and neutron stars is given. In a recent paper [15], the generation of a magnetic field by TMI in the surface layers (hot plasma) of massive stars 
was considered. Such generation is possible in the upper layers of the atmosphere of hot stars, where deviations from local thermodynamic equilibrium form a region with an inverse temperature gradient. In [15], the case of the generation of only small-scale magnetic fields with horizontal wavelengths $\lambda=2 \pi / k_{x}$ much smaller than the characteristic scale $L$ of unperturbed quantities $\lambda \ll L$ was considered. Thus, TMI is an alternative mechanism of the origin of magnetic fields in various astrophysical objects along with the existing theories of the turbulent [2-10] and convective dynamo [16].

In some recent works [17-19], the TMI in nonuniformly rotating plasma media (hot galactic disks, accretion disks) in an external axial magnetic field was considered. These papers present an analysis of the linear stability of ionized hot disks with a temperature gradient and an external axial magnetic field. Earlier, Gurevich and Helmont [20] studied the destabilizing effect of the temperature gradient on the propagation of Alfven waves in astrophysical plasma in the absence of a hydrodynamic motion. As shown in [17-19], the hydromagnetic and thermomagnetic effects associated with the Nernst effect can lead to the amplification of waves and make disks unstable. The regimes under which both thermomagnetic and magnetorotational instabilities (MRI) can operate were discussed. MRI arises when a weak axial magnetic field destabilizes the azimuthal differential rotation of the plasma, and when the condition $d \Omega^{2} / d R<0$ for the case of a nondissipative plasma is satisfied [21]. Since this condition is also satisfied for Keplerian flows $\Omega \sim R^{-3 / 2}$, the MRI is the most likely source of turbulence in accretion disks [22]. It was noted in [19] that even in the absence of MRI, TMI due to the Nernst effect is a good candidate for ensuring the onset of turbulence in disks.

Unlike papers [15, 17-19], in this paper we studied the spontaneous generation of a magnetic field by TMI in a nonuniformly rotating layer of an electrically conducting fluid in the presence of an external constant axial magnetic field. Here TMI is due to the collinear temperature gradient and the gradient of the thermoelectromotive force coefficient. This work is a continuation of the research begun in [23], where the problem of the stability of an electrically conducting fluid between two rotating cylinders (Couette flow) and the Rayleigh-Benard problem in an external constant magnetic field were considered. In contrast to [23], we studied the influence of TM effects on convective processes, as well as the weakly nonlinear evolution of the toroidal magnetic field generated by TMI. The work consists of the following sections. Section I (Introduction) gives a brief overview of the main problems of modern dynamo theory. In Section II, the basic equations of magnetohydrodynamics taking into account thermomagnetic phenomena in the Boussinesq approximation are obtained. These equations describe the generation of magnetic fields in a nonuniformly rotating electrically conducting fluid in a constant magnetic field. In Section III, the stationary magnetic convection (the Rayleigh-Benard problem) in a nonuniformly rotating liquid layer is considered, where a toroi- dal magnetic field is generated due to the temperature difference and specific thermopower at the layer boundaries. In Section IV, we investigated the weakly nonlinear stage of a stationary inhomogeneously rotating magnetic convection taking into account TM effects. We obtained the nonlinear Ginzburg-Landau equation applying the method of perturbation theory in the small parameter of the supercriticality of the Rayleigh number $\epsilon=\sqrt{\left(\mathrm{Ra}-\mathrm{Ra}_{c}\right) / \mathrm{Ra}_{c}}$. In the last Section V (Conclusion) we presented the main results obtained in the work.

\section{STATEMENT OF THE PROBLEM AND EQUATIONS OF THE EVOLUTION OF SMALL PERTURBATIONS}

Let us consider the following problem statement. Let us asume that a nonuniformly rotating electrically conducting fluid (for example, liquid metal or plasma) is in a constant gravitational $\mathbf{g}$ and magnetic field $\mathbf{B}_{0}$ at a constant vertical temperature gradient $\nabla T_{0}=$ const $=$ $-A \mathbf{e}(A>0)$ is a constant gradient, e is a unit vector directed vertically upward along the axis) and a gradient of the thermoelectromotive force coefficient $\nabla \alpha \| \mathbf{e}$. In the model considered here, we assume that the gradient of the specific thermoelectric power is associated with the inhomogeneity of the chemical composition of the conducting liquid. In this model, we assume that the gradient of the thermoelectromotive force coefficient $\nabla \alpha$ is associated with the inhomogeneity of the chemical composition of the conducting fluid. It is known that expressions for Ohm's law and heat flux $\mathbf{q}$ in the presence of a magnetic field $\mathbf{B}$ and a temperature gradient $\nabla T$ are modified taking into account thermomagnetic phenomena [24]:

$$
\begin{aligned}
& \mathbf{E}+[\mathbf{V} \times \mathbf{B}]=\frac{\mathbf{j}}{\sigma}+\alpha \nabla T+\mathcal{R}[\mathbf{B} \times \mathbf{j}]+\mathcal{N}[\mathbf{B} \times \nabla T], \\
& \mathbf{q}-\varphi \mathbf{j}=-\kappa \nabla T+\alpha T \mathbf{j}+\mathcal{N} T[\mathbf{B} \times \mathbf{j}]+\mathcal{L}[\mathbf{B} \times \nabla T],
\end{aligned}
$$

where $\mathcal{R}, \mathcal{N}, \mathcal{L}$ are the Hall, Nernst, and LeducRighi coefficients, respectively; $\varphi$ - electrical potential. In expressions (1)-(2), we neglected the anisotropy of the coefficients of electrical conductivity $\sigma_{\|} \approx$ $\sigma_{\perp}=\sigma$, thermal conductivity $\kappa_{\|} \approx \kappa_{\perp}=\kappa$, and thermoelectromotive force $\alpha_{\|} \approx \alpha_{\perp}=\alpha$ due to the weakness of the external magnetic field $\mathbf{B}_{0}$ because the parameter is small $\beta=B_{0}^{2} / 2 \mu P_{0} \ll 1$ ( $P_{0}$ is the stationary pressure of the fluid, $\mu$ is the coefficient of magnetic permeability). By applying the operation rot to Ohm's law (1), we obtain the equation for the magnetic field induction B. After the substitution of expression (2) into the heat balance equation

$$
\rho_{0} c_{p} \frac{d T}{d t}=-\operatorname{div} \mathbf{q}
$$

let us write the equations of magnetohydrodynamics for a viscous incompressible fluid in the Boussinesq approximation taking into account thermomagnetic phenomena:

$$
\begin{aligned}
\frac{\partial \mathbf{V}}{\partial t} & +(\mathbf{V} \cdot \nabla) \mathbf{V}=-\frac{1}{\rho_{0}} \nabla\left(P+\frac{\mathbf{B}^{2}}{2 \mu}\right)+\frac{1}{\rho_{0} \mu}(\mathbf{B} \cdot \nabla) \mathbf{B} \\
& +\mathbf{e} g \beta_{T} T+\nu \nabla^{2} \mathbf{V}
\end{aligned}
$$




$$
\begin{aligned}
\frac{\partial \mathbf{B}}{\partial t} & +(\mathbf{V} \cdot \nabla) \mathbf{B}-(\mathbf{B} \cdot \nabla) \mathbf{V}=\eta \nabla^{2} \mathbf{B}-[\nabla \alpha \times \nabla T] \\
& -\frac{\mathcal{R}}{\mu} \operatorname{rot}[\mathbf{B} \times \operatorname{rot} \mathbf{B}]-\mathcal{N} \operatorname{rot}[\mathbf{B} \times \nabla T] \\
\frac{\partial T}{\partial t} & +(\mathbf{V} \cdot \nabla) T=-\frac{1}{\rho_{0} c_{p}} \operatorname{div}\left(-\kappa \nabla T+\frac{\mathbf{j}^{2}}{\sigma}+\alpha T \mathbf{j}\right. \\
& +\mathcal{N} T[\mathbf{B} \times \mathbf{j}]+\mathcal{L}[\mathbf{B} \times \nabla T])
\end{aligned}
$$

$$
\operatorname{div} \mathbf{B}=0, \quad \operatorname{div} \mathbf{V}=0,
$$

where $\beta_{T}$ is the coefficient of thermal expansion, $\rho_{0}=$ const is the density of the medium, $\nu$ is the coefficient of kinematic viscosity, $\eta=1 / \mu \sigma$ is the coefficient of magnetic viscosity. Equation (4) contains a source of excitation of a magnetic field of non-electromagnetic nature $[\nabla \alpha \times \nabla T]$, which is an analog of the "battery" Biermann-Schlüter effect in the plasma. The drift of the lines of force of the magnetic field in equation (4) is associated not only with the movement of the fluid $\mathbf{V}$ but also with the heat flux where the rate of thermal drift is equal to: $\mathbf{V}_{T}=\mathcal{N} \nabla T$. The drift of the magnetic field due to the Nernst effect contributes to its penetration to a large area of the medium. Let us estimate the excited magnetic field in the stationary regime without taking into account the drift of the field and the Hall effect. Then from (4) for the $\phi$ component of the (toroidal) magnetic field, we obtain: $B_{\phi}^{\max } \approx \alpha T \mu \sigma\left(L_{B} / L_{\alpha}\right)$, where $L_{B}$ is the characteristic scale of the excited magnetic field, $L_{\alpha}$ is the characteristic scale of the medium inhomogeneity. Substituting the values of the parameters of the fluid Earth's core: $\alpha T \cong 10^{-2} \mathrm{~V}$ (at temperature $T \cong 1000 \mathrm{~K}$ ) [12], $\mu=4 \pi \cdot 10^{-7} \mathrm{~V} \cdot \mathrm{s} / \mathrm{A} \cdot \mathrm{m}, \sigma=3 \cdot 10^{5}(\mathrm{~V} \cdot \mathrm{m} / \mathrm{A})^{-1}[6]$ for the ratio of scales $\left(L_{B} / L_{\alpha}\right)=10^{2}$, we obtain an estimated value of the toroidal magnetic field of the Earth's core $B_{\phi}^{\max } \cong 10^{-1} \mathrm{~T}$, which coincides in order of magnitude with the data from monograph [25].
Let us investigate the possibility of generating a magnetic field as a consequence of the development of TM instability by presenting all quantities in equations (3)-(6) as the sum of the stationary and perturbed parts $\mathbf{V}=\mathbf{V}_{0}+\mathbf{u}, \mathbf{B}=\mathbf{B}_{0}+\mathbf{b}, P=P_{0}+p, T=T_{0}+\theta$. Here we assume that the stationary rotation velocity of the fluid has an azimuthal direction $\mathbf{V}_{0}=R \Omega(R) \mathbf{e}_{\phi}$. The angular velocity of rotation $\Omega(R)$ is directed vertically upward along the axis $O Z$. The homogeneous (constant) magnetic field $\mathbf{B}_{0} \| \boldsymbol{\Omega}$ is also directed along the axis $\mathrm{OZ}$ : $\mathbf{B}_{0}=\left(0,0, B_{0}\right)$. Further, the magnetic field $\mathbf{B}_{0}$ will be called axial in the cylindrical coordinate system $(R, \phi, z)$. The stationary state of the system of equations (3)-(6) is described by the following equations:

$$
\begin{gathered}
\Omega^{2} R=\frac{1}{\rho_{0}} \frac{d P_{0}}{d R}, \quad \frac{1}{\rho_{0}} \frac{d P_{0}}{d z}=g \beta_{T} T_{0}, \quad \frac{d^{2} T_{0}}{d z^{2}}=0 . \\
B_{0} \frac{d}{d z} \Omega(R) R=\left[\nabla \alpha \times \nabla T_{0}\right]_{\phi}=0 .
\end{gathered}
$$

Equations (8) show that centrifugal equilibrium is established in the radial direction, and hydrostatic equilibrium in the vertical direction. From equation (8) it follows that the thermoelectromotive coefficient $\alpha$ has a constant value in the radial direction: $d \alpha / d R=0$, then it can have a dependence on the coordinates $(\phi, z)$. If we consider the distribution of the chemical composition of the medium to be axisymmetric, then the condition is satisfied: $d \alpha / d z \neq 0$. In this case, the condition of collinearity of vectors is also satisfied $\left[\nabla \alpha \times \nabla T_{0}\right]=0$, and the gradients $\nabla \alpha$ and $\nabla T_{0}$ can be both parallel to each other $\nabla \alpha \uparrow \uparrow \nabla T_{0}$ and antiparallel: $\nabla \alpha \uparrow \downarrow \nabla T_{0}$. The evolution equations for the perturbed quantities $(\mathbf{u}, \mathbf{b}, p, \theta)$ against the background of a stationary state take the following form:

$$
\begin{aligned}
\frac{\partial \mathbf{u}}{\partial t}+\left(\mathbf{V}_{0} \cdot \nabla\right) \mathbf{u}+(\mathbf{u} \cdot \nabla) \mathbf{V}_{0} & =-\frac{1}{\rho_{0}} \nabla\left(p+\frac{1}{\mu} \mathbf{B}_{0} \mathbf{b}+\frac{1}{2 \mu} \mathbf{b}^{2}\right) \\
& +\frac{1}{\rho_{0} \mu}\left(\mathbf{B}_{0} \cdot \nabla\right) \mathbf{b}+g \beta_{T} \theta \mathbf{e}+\nu \nabla^{2} \mathbf{u}+R_{\mathrm{NL}}^{(1)}, \\
\frac{\partial \mathbf{b}}{\partial t}+\left(\mathbf{V}_{0} \cdot \nabla\right) \mathbf{b}-\left(\mathbf{B}_{0} \cdot \nabla\right) \mathbf{u}-(\mathbf{b} \cdot \nabla) \mathbf{V}_{0} & =\eta \nabla^{2} \mathbf{b}-[\nabla \alpha \times \nabla \theta]-\frac{\mathcal{R}}{\mu} \operatorname{rot}\left[\mathbf{B}_{0} \times \operatorname{rot} \mathbf{b}\right] \\
& -\mathcal{N} \operatorname{rot}\left(\left[\mathbf{B}_{0} \times \nabla \theta\right]+\left[\mathbf{b} \times \nabla T_{0}\right]\right)+R_{\mathrm{NL}}^{(2)}, \\
\frac{\partial \theta}{\partial t}+\left(\mathbf{V}_{0} \cdot \nabla\right) \theta+(\mathbf{u} \cdot \nabla) T_{0} & =\chi \nabla^{2} \theta-\frac{\alpha T_{0}}{\rho_{0} c_{p} \mu}\left(\mathbf{K}_{\alpha}+\mathbf{K}_{T}\right) \operatorname{rot} \mathbf{b}-\frac{\mathcal{N} T_{0}}{\rho_{0} c_{p} \mu} \operatorname{div}\left[\mathbf{B}_{0} \times \operatorname{rot} \mathbf{b}\right] \\
& -\chi \wedge \operatorname{div}\left(\left[\mathbf{b} \times \nabla T_{0}\right]+\left[\mathbf{B}_{0} \times \nabla \theta\right]\right)+R_{\mathrm{NL}}^{(3)}, \\
\operatorname{div} \mathbf{b} & =0, \quad \operatorname{div} \mathbf{u}=0,
\end{aligned}
$$


where the nonlinear terms $R_{\mathrm{NL}}^{(1)}, R_{\mathrm{NL}}^{(2)}, R_{\mathrm{NL}}^{(3)}$ are equal respectively:

$$
\begin{aligned}
R_{\mathrm{NL}}^{(1)} & =-(\mathbf{u} \cdot \nabla) \mathbf{u}+\frac{1}{\rho_{0} \mu}(\mathbf{b} \cdot \nabla) \mathbf{b}, \\
R_{\mathrm{NL}}^{(2)} & =(\mathbf{b} \cdot \nabla) \mathbf{u}-(\mathbf{u} \cdot \nabla) \mathbf{b}-\frac{\mathcal{R}}{\mu} \operatorname{rot}[\mathbf{b} \times \operatorname{rot} \mathbf{b}]-\mathcal{N} \operatorname{rot}[\mathbf{b} \times \nabla \theta], \\
R_{\mathrm{NL}}^{(3)} & =-(\mathbf{u} \cdot \nabla) \theta-\frac{1}{\rho_{0} c_{p} \mu}(\alpha \nabla \theta+\theta \nabla \alpha) \operatorname{rot} \mathbf{b}-\frac{\mathcal{N}}{\rho_{0} c_{p} \mu}\left(\nabla T_{0} \cdot[\mathbf{b} \times \operatorname{rot} \mathbf{b}]\right. \\
& \left.+\nabla \theta \cdot\left[\left(\mathbf{B}_{0}+\mathbf{b}\right) \times \operatorname{rot} \mathbf{b}\right]+\left(T_{0}+\theta\right) \operatorname{div}[\mathbf{b} \times \operatorname{rot} \mathbf{b}]+\theta \operatorname{div}\left[\mathbf{B}_{0} \times \operatorname{rot} \mathbf{b}\right]\right)-\chi_{\wedge} \operatorname{div}[\mathbf{b} \times \nabla \theta] .
\end{aligned}
$$

In equations (9) new introduced designations $\mathbf{K}_{\alpha}=$ $\nabla \alpha / \alpha, \mathbf{K}_{T}=\nabla T_{0} / T_{0}$ are scales of inhomogeneity of the medium $L_{\alpha} \cong\left|\mathbf{K}_{\alpha}\right|^{-1}, L_{T} \cong\left|\mathbf{K}_{T}\right|^{-1}, \chi_{\wedge}=$ $\mathcal{L} / \rho_{0} c_{p}$ is the skewed coefficient of thermal diffusivity. The system of equations (9) is quite complicated for a complete analysis. Next, we consider the evolution of small perturbations by linearizing Eqs. (9). Then we can neglect nonlinear terms. The generation of magnetic fields arises due to the effects associated with the inhomogeneity of the thermoelectromotive force coefficient and magnetization of the heat flux (Leduc-Righi effect). Thus, in equation (9), we restrict ourselves to only taking these effects into account. We will consider the evolution of axisymmetric perturbations, i.e. independent of the azimuthal angle $\phi(\partial / \partial \phi=0)$, and we apply the local WKB method for equations (9) for small perturbations that depend on radial coordinates $R$. For this purpose, we expand all quantities in a Taylor series in the vicinity of fixed points $R_{0}$ leaving the terms of order zero in local coordinates $\widetilde{R}=R-R_{0}$. As a result, we obtain a system of differential equations with constant coefficients. All perturbations in this system of equations are represented in the form of plane waves

$$
(\mathbf{u}, \mathbf{b}, \theta, \widetilde{p})=(\mathbf{U}(z), \mathbf{H}(z), \Theta(z), \widetilde{P}(z)) \exp (\gamma t+i k \widetilde{R}) .
$$

As a result of simple but cumbersome mathematical operations, these equations are reduced to one differential equation for $U_{z}$ :

$$
\begin{aligned}
& {\left[\widehat{a}_{11}\left(\widehat{a}_{22} \widehat{a}_{33}-\widehat{a}_{23} \widehat{a}_{32}\right)+\widehat{a}_{12}\left(\widehat{a}_{23} \widehat{a}_{31}-\widehat{a}_{21} \widehat{a}_{33}\right)\right.} \\
& \left.+\widehat{a}_{13}\left(\widehat{a}_{21} \widehat{a}_{32}-\widehat{a}_{31} \widehat{a}_{22}\right)\right] U_{z}=0,
\end{aligned}
$$

where $\operatorname{Pr}=\nu / \chi$ is the Prandtl number, $\operatorname{Pm}=\nu / \eta$ is the Prandtl magnetic number, Ta $=\frac{4 \Omega_{0}^{2} h^{4}}{\nu^{2}}$ is the Taylor number, $\mathrm{Ha}=\frac{B_{0} h}{\sqrt{\rho_{0} \mu \nu \eta}}$ is the Hartman number, $\mathrm{Ra}=\frac{g \beta_{T}(\Delta T) h^{3}}{\nu \chi}$ is the Rayleigh number, $\mathrm{R}_{\alpha}=\frac{\Delta \alpha \Delta T}{\eta B_{0}}$ is the thermoelectromotive force number, dimensionless parameter:

$$
q_{\alpha}=\frac{\alpha T_{0} B_{0}}{\rho_{0} c_{p} \mu \chi(\Delta T)}\left[\left(\frac{\mu \mathcal{L}}{\alpha}+1\right) \frac{\Delta T}{T_{0}}+\frac{\Delta \alpha}{\alpha}\right]
$$

is associated with the influence of the thermoelectromotive force and Leduc-Righi effects on the heat transfer process. We use the Chandrasekhar numbers $\mathrm{Q}=\mathrm{Ha}^{2}$ and $\widetilde{\mathrm{Q}}=\mathrm{QPm}^{-1} \mathrm{Pr}$ instead of the Hartmann number Ha for convenience. Equation (11) is supplemented with boundary conditions only in the $z$-direction

$$
U_{z}=\frac{d^{2} U_{z}}{d z^{2}}=0 \quad \text { at } \quad z=0 \quad \text { and } \quad z=1 .
$$

Equation (11) with boundary condition (12) describes convective phenomena in a thin layer of a nonuniformly rotating magnetized fluid with thermomagnetic effects.

\section{GENERATION OF A MAGNETIC FIELD BY THERMOMAGNETIC EFFECTS IN A THIN LAYER OF A NONUNIFORMLY ROTATING FLUID}

Let us consider a stationary flow of a nonuniformly rotating incompressible viscous electrically conductive fluid, which is modeled by the Couette-Taylor flow enclosed between two rotating cylinders with an angular velocity of rotation $\Omega(R)$ :

$$
\Omega(R)=\frac{\Omega_{2} R_{2}^{2}-\Omega_{1} R_{1}^{2}}{R_{2}^{2}-R_{1}^{2}}+\frac{\left(\Omega_{1}-\Omega_{2}\right) R_{1}^{2} R_{2}^{2}}{R^{2}\left(R_{2}^{2}-R_{1}^{2}\right)},
$$

where $R_{1}=R_{\text {in }}, R_{2}=R_{\text {out }}, \Omega_{1}=\Omega_{\text {in }}, \Omega_{2}=\Omega_{\text {out }}$ are radius and angular velocity of rotation of the inner and outer cylinders, respectively. The choice of this type of flow is due to the possibility of realization of the theory developed here in laboratory experiments. The height of the cylinders corresponds to a liquid layer of finite thickness $h$ under the condition $h \ll\left(R_{\text {out }}-R_{\text {in }}\right)$. The geometry of the problem is shown in Fig. 1. On the lower plane of the layer, a higher temperature $T_{\mathrm{d}}$ is supported than on the upper plane $T_{\mathrm{u}}: T_{\mathrm{d}}>T_{\mathrm{u}}$ the heating from below. The thermoelectromotive force coefficient $\alpha_{\mathrm{d}}$ on the lower (hot) plane is less than on the upper (cold) plane $\alpha_{\mathrm{u}}$ : $\alpha_{\mathrm{d}}<\alpha_{\mathrm{u}}$. This situation is quite possible if we take into account the dependence of the thermoelectromotive force coefficient on temperature $\alpha \sim \psi / T_{0}$ ( $\psi$ is the chemical potential) [24]. A spatially 
inhomogeneous distribution inside a layer $T_{0}(z)$ and $\alpha(z)$ can be represented as a linear dependence on $z$ :

$T_{0}(z)=T_{\mathrm{d}}-\frac{\Delta T}{h} \cdot z, \quad \Delta T=T_{\mathrm{d}}-T_{\mathrm{u}}, \quad \alpha(z)=\alpha_{\mathrm{d}}+\frac{\Delta \alpha}{h} \cdot z$,

$\Delta \alpha=\alpha_{\mathrm{u}}-\alpha_{\mathrm{d}}$

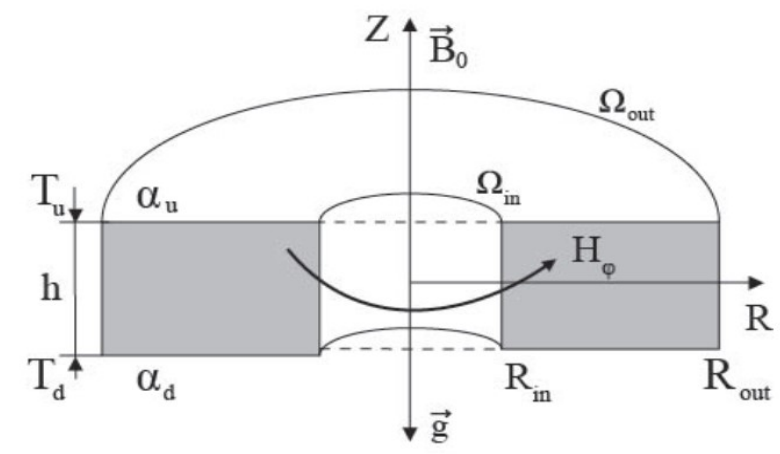

Fig. 1. An electrically conductive fluid fills in a layer between two rotating cylinders with angular velocities $\Omega_{\text {in }}$ and $\Omega_{\text {out }}$, respectively. The lower surface of the layer has the temperature $T_{\mathrm{d}}$ and the thermoelectromotive force coefficient $\alpha_{\mathrm{d}}$, and the upper surface has $T_{\mathrm{u}}$ and $\alpha_{\mathrm{u}}: T_{\mathrm{d}}>T_{\mathrm{u}}, \alpha_{\mathrm{d}}<\alpha_{\mathrm{u}}$. The generated magnetic field has an azimuthal direction $H_{\phi}$.

As is known [26], the temperature difference at the boundaries of the layer in the gravity field $\mathrm{g}$ leads to a violation of mechanical equilibrium in the system. In this case, the convective instability develops and convective cells are formed. Temperature perturbations acting in the radial direction lead to the appearance of a vortex thermal current due to the difference in the values of the thermoelectromotive force coefficient $\Delta \alpha(z)$ at the layer boundaries. This current induces disturbances of the azimuthal (toroidal) magnetic field $H_{\phi}$ (see Fig. 1), which influences the heat transfer regime. The excited magnetic field $H_{\phi}$ creates heat fluxes directed perpendicular to the field itself and the temperature gradient. Thus, positive feedback is established: newly arising heat fluxes create a vortex thermoelectromotive power, which enhances magnetic field disturbances $H_{\phi}$. Naturally, the thermomagnetic instability will affect the development of convective instability. We will consider this issue in the current section.

\section{A. Dispersion equation for TM perturbations}

For simplicity, the solution of equation (11) with boundary condition (12) will be sought in the form of a single-mode approximation

$$
U_{z}=W_{0} \sin \pi z,
$$

where $W_{0}$ is a constant amplitude. Substituting (13) into (11) and integrating over the layer thickness $z=(0,1)$, we obtain the dispersion equation

$$
\mathrm{Ra}=\left(\operatorname{Ra}^{(0)}-\mathrm{Ra}^{(\mathrm{TM})}\right)\left(1-\frac{k^{2} q_{\alpha} R_{\alpha}}{\Gamma_{\eta} \Gamma_{\chi}}\right) \Delta^{-1},
$$

where $\mathrm{Ra}^{(0)}$ is the contribution to the dispersion equation without taking into account TM effects, obtained in [23]:

$$
\begin{aligned}
& \operatorname{Ra}^{(0)}=\frac{\Gamma_{\chi}\left(a^{2} \Gamma_{A}^{4}+\pi^{2} \mathrm{Ta}(1+\mathrm{Ro}) \Gamma_{\eta}^{2}+\pi^{4} \mathrm{Ha}^{2} \mathrm{TaRoPm}\right)}{k^{2} \Gamma_{\eta} \Gamma_{A}^{2}}, \\
& \operatorname{Ra}^{(\mathrm{TM})}=q_{\alpha} R_{\alpha} \frac{a^{2}\left(\gamma+a^{2}\right)}{\Gamma_{\eta}}+q_{\alpha} R_{\alpha} \frac{\pi^{2} \mathrm{Ta}}{\Gamma_{A}^{2}}(1+\mathrm{Ro})+R_{\alpha} \frac{\pi^{2} \mathrm{Q}}{\Gamma_{A}^{2}} \operatorname{PmPr}^{-1} \sqrt{\mathrm{Ta}}, \\
& \Delta=\left(1-\frac{q_{\alpha}}{\Gamma_{\eta}^{2}} \pi^{2} \operatorname{Pm}^{2} \operatorname{Pr}^{-1} \operatorname{Ro} \sqrt{\mathrm{Ta}}\right)\left(1-\frac{q_{\alpha} R_{\alpha}}{\Gamma_{\chi} \Gamma_{A}^{2}} k^{2}\left(\gamma+a^{2}\right)\right)+\frac{q_{\alpha} \pi^{2} \operatorname{PmPr}^{-1}}{\Gamma_{\eta}^{2} \Gamma_{\chi} \Gamma_{A}^{2}} \\
& \times\left(\sqrt{\mathrm{Ta}}(1+\operatorname{Ro}) \Gamma_{\eta}\left(\Gamma_{\eta} \Gamma_{\chi}-k^{2} q_{\alpha} R_{\alpha}\right)+\pi^{2} \mathrm{QPmRo} \sqrt{\mathrm{Ta}} \Gamma_{\chi}\right)-\frac{q_{\alpha} R_{\alpha} \pi^{2} k^{2} \mathrm{Q}}{\Gamma_{\eta} \Gamma_{\chi} \Gamma_{A}^{2}},
\end{aligned}
$$

where the new notation is introduced

$$
\begin{gathered}
\Gamma_{A}^{2}=\left(\gamma+a^{2}\right)\left(\gamma \mathrm{Pm}+a^{2}\right)+\pi^{2} \mathrm{Ha}^{2}, \quad \Gamma_{\chi}=\gamma \operatorname{Pr}+a^{2}, \\
\Gamma_{\eta}=\gamma \mathrm{Pm}+a^{2}, \quad a^{2}=\pi^{2}+k^{2} .
\end{gathered}
$$

In the absence of thermal processes, MRI arises in a nonuniformly rotating layer of an electrically conductive fluid in a constant magnetic field. In this case, equation (14) coincides with the dispersion equation for the standard MRI (SMRI) taking into account dissipative processes [27]. The threshold value of the hydrodynamic Rossby number Ro is determined using the condition $\gamma=0$ and has the form:

$$
\mathrm{Ro}_{\mathrm{cr}}=-\frac{a^{2}\left(a^{4}+\pi^{2} \mathrm{Ha}^{2}\right)^{2}+\pi^{2} a^{4} \mathrm{Ta}}{\pi^{2} \mathrm{Ta}\left(a^{4}+\pi^{2} \mathrm{Ha}^{2} \mathrm{Pm}\right)} .
$$


When transforming to dimensional variables

$$
\begin{gathered}
\frac{\pi^{2} \mathrm{Ha}^{2}}{a^{4}} \rightarrow \frac{\omega_{A}^{2}}{\omega_{\nu} \omega_{\eta}}, \frac{\pi^{2} \mathrm{Ha}^{2} \mathrm{Pm}}{a^{4}} \rightarrow \frac{\omega_{A}^{2}}{\omega_{\eta}^{2}}, \\
\frac{\mathrm{Ta}}{a^{4}} \rightarrow \frac{4 \Omega^{2}}{\omega_{\nu}^{2}}, \frac{\pi^{2}}{a^{2}} \rightarrow \xi^{2}
\end{gathered}
$$

the expression for $\mathrm{Ro}_{\mathrm{cr}}$ is found [27]

$$
\operatorname{Ro}_{\mathrm{cr}}=-\frac{\left(\omega_{A}^{2}+\omega_{\nu} \omega_{\eta}\right)^{2}+4 \xi^{2} \Omega^{2} \omega_{\eta}^{2}}{4 \Omega^{2} \xi^{2}\left(\omega_{A}^{2}+\omega_{\eta}^{2}\right)}
$$

The criterion for MRI appearance is the condition imposed on the angular velocity profile $\Omega(R)$ of the rotating fluid, i.e., Rossby number $\mathrm{Ro}>\mathrm{Ro}_{\mathrm{cr}}$. Let us now analyze a more general case when there is the heati$\mathrm{ng}$ of the fluid layer $\mathrm{Ra} \neq 0$ and its nonuniform rotation Ro $\neq 0$ taking into account the thermomagnetic effects.

\section{B. Stationary convection regime}

Obviously, for the stationary convection mode, the increment $\gamma$ is zero $(\gamma=0)$, therefore, from formula (14) we can find the critical value of the Rayleigh number $\mathrm{Ra}_{\mathrm{st}}$ for stationary convection:

$$
\mathrm{Ra}_{\mathrm{st}}=\left[\mathrm{Ra}_{\mathrm{st}}^{(0)}-a^{2} q_{\alpha} R_{\alpha}-\frac{\pi^{2} R_{\alpha}}{a^{4}+\pi^{2} \mathrm{Q}}\left(\operatorname{Ta}(1+\mathrm{Ro}) q_{\alpha}+\widetilde{\mathrm{Q}} \sqrt{\mathrm{Ta}}\right)\right]\left[1+\frac{q_{\alpha} \pi^{2} \operatorname{PmPr}^{-1} \sqrt{\operatorname{Ta}}(1+\operatorname{Ro}(1-\operatorname{Pm}))}{a^{4}+\pi^{2} \mathrm{Q}}\right]^{-1}
$$

where

$$
\mathrm{Ra}_{\mathrm{st}}^{(0)}=\frac{a^{6}}{k^{2}}+\frac{a^{2} \pi^{2} \mathrm{Q}}{k^{2}}+\frac{\pi^{2} \mathrm{Ta}}{k^{2}} \cdot \frac{a^{4}+\operatorname{Ro}\left(a^{4}+\pi^{2} \mathrm{QPm}\right)}{a^{4}+\pi^{2} \mathrm{Q}} .
$$

The minimum value of the critical Rayleigh number is found from the condition $\partial \mathrm{Ra}_{\mathrm{st}} / \partial k=0$ and corresponds to the wavenumbers $k=k_{c}$ that satisfy the following equation:

$$
\begin{aligned}
& \left(1+\frac{q_{\alpha} \pi^{2} \operatorname{PmPr}^{-1} \sqrt{\mathrm{Ta}}(1+\mathrm{Ro}(1-\mathrm{Pm}))}{\left(\pi^{2}+k_{c}^{2}\right)^{2}+\pi^{2} \mathrm{Q}}\right) \\
& \times\left(\mathrm{M}\left(k_{c}\right)-\mathrm{R}_{\mathrm{TM}} \cdot \frac{k_{c}^{3}\left(\pi^{2}+2 k_{c}^{2}\right)}{\left(\pi^{2}+k_{c}^{2}\right)}+\mathrm{R}_{\mathrm{TM}} \cdot \frac{2 \pi^{2} k_{c}^{3}\left(\mathrm{Ta}\left(1+\mathrm{Ro}^{2}\right) \mathrm{R}_{\mathrm{TM}}+R_{\alpha} \widetilde{\mathrm{Q}} \sqrt{\mathrm{Ta}}\right.}{\left(\pi^{2}+k_{c}^{2}\right)\left(\left(\pi^{2}+k_{c}^{2}\right)^{2}+\pi^{2} \mathrm{Q}\right)^{2}}\right) \\
& +\left(\frac{\left(\pi^{2}+k_{c}^{2}\right)^{3}}{k_{c}^{2}}-\mathrm{R}_{\mathrm{TM}} \cdot k_{c}^{2}\left(\pi^{2}+k_{c}^{2}\right)+\frac{\pi^{2}+k_{c}^{2}}{k_{c}^{2}} \cdot \pi^{2} \mathrm{Q}\right. \\
& \left.+\frac{\pi^{2} \mathrm{Ta}\left(1+\mathrm{Ro}^{2}\right)\left(\left(\pi^{2}+k_{c}^{2}\right)^{2}-k_{c}^{2} \mathrm{R}_{\mathrm{TM}}\right)+\pi^{4} \mathrm{QPmRoTa}-k_{c}^{2} \pi^{2} R_{\alpha} \widetilde{\mathrm{Q}} \sqrt{\mathrm{Ta}}}{k_{c}^{2}\left(\left(\pi^{2}+k_{c}^{2}\right)^{2}+\pi^{2} \mathrm{Q}\right)^{2}}\right) \\
& \times \frac{2 k_{c}^{3} \pi^{2} q_{\alpha} \operatorname{PmPr} \operatorname{Pr}^{-1} \sqrt{\mathrm{Ta}}\left(1+\mathrm{Ro}^{2}(1-\mathrm{Pm})\right)}{\left(\pi^{2}+k_{c}^{2}\right)\left(\left(\pi^{2}+k_{c}^{2}\right)^{2}+\pi^{2} \mathrm{Q}\right)^{2}}=0,
\end{aligned}
$$

where $\mathrm{R}_{\mathrm{TM}}=q_{\alpha} R_{\alpha}$ is a dimensionless parameter depending on the temperature the gradient and gradient of the thermoelectromotive force coefficient. In the limiting case, when TM effects are absent, this equation coincides with the result of [23]:

$$
\begin{aligned}
\mathrm{M}\left(k_{c}\right) & =\frac{2 k_{c}^{2}-\pi^{2}}{k_{c}}-\frac{\pi^{4} \mathrm{Q}}{k_{c}\left(\pi^{2}+k_{c}^{2}\right)^{2}}+\frac{2 \pi^{2} k_{c} \mathrm{Ta}(1+\mathrm{Ro})}{\left(\pi^{2}+k_{c}^{2}\right)\left(\left(\pi^{2}+k_{c}^{2}\right)^{2}+\pi^{2} \mathrm{Q}\right)} \\
& -\frac{\pi^{2} \mathrm{Ta}\left(\left(\pi^{2}+k_{c}^{2}\right)^{2}+\pi^{2} \mathrm{Q}+2 k_{c}^{2}\left(\pi^{2}+k_{c}^{2}\right)\right)}{k_{c}\left(\left(\pi^{2}+k_{c}^{2}\right)^{2}+\pi^{2} \mathrm{Q}\right)^{2}} \\
& -\frac{\pi^{2} \operatorname{TaRo}\left(\left(\pi^{2}+k_{c}^{2}\right)^{2}+\pi^{2} \mathrm{QPm}\right)\left(\left(\pi^{2}+k_{c}^{2}\right)^{2}+\pi^{2} \mathrm{Q}+2 k_{c}^{2}\left(\pi^{2}+k_{c}^{2}\right)\right)}{k_{c}\left(\pi^{2}+k_{c}^{2}\right)^{2}\left(\left(\pi^{2}+k_{c}^{2}\right)^{2}+\pi^{2} \mathrm{Q}\right)^{2}}=0 .
\end{aligned}
$$


Let us consider some limiting cases.

1. In the absence of rotation ( $\mathrm{Ta}=0, \mathrm{Ro}=0)$ and magnetic field $\left(\mathbf{B}_{0}=0\right)$, from expression (15) we found

$$
\mathrm{Ra}_{\mathrm{st}}=\frac{\left(k^{2}+\pi^{2}\right)^{3}}{k^{2}}-\mathrm{R}_{\mathrm{TM}}\left(k^{2}+\pi^{2}\right) .
$$

If the gradient of the thermoelectromotive force coefficient is zero (the medium is chemically homogeneous), then the well-known result follows from (16) $\mathrm{Ra}_{\mathrm{st}}^{\min }=\left(k^{2}+\pi^{2}\right)^{3} / k^{2}$. Here, the minimum value of the critical Rayleigh number $\mathrm{Ra}_{\mathrm{st}}=$ $27 \pi^{4} / 4$ is reached at wavenumber $k_{c}=\pi / \sqrt{2}$ [26]. The minimum value of the critical

Rayleigh number $\mathrm{Ra}_{\mathrm{st}}^{\min }$ is calculated by the formula (16) at $k=k_{c}$, which satisfies the following relation

$$
2\left(\frac{k_{c}}{\pi}\right)^{6}+3\left(\frac{k_{c}}{\pi}\right)^{4}=1+\left(\frac{k_{c}^{4}}{\pi^{6}}\right) \mathrm{R}_{\mathrm{TM}} .
$$

The numerical value $\mathrm{Ra}_{\mathrm{st}}^{\min }$ in Fig. 2 corresponds to a point on the neutral curve separating the regions of stable and unstable disturbances. It can be seen here that with an increase in the coefficient the minimum value of the critical Rayleigh number decreases, i.e. the threshold for the development of instability decreases. A numerical estimate of the coefficient $R_{\mathrm{TM}}$ was carried out for the physical parameters of the Earth's core: $\rho_{0} \approx 7 \cdot 10^{3} \mathrm{~kg} / \mathrm{m}^{3}$ is the density of molten iron, $c_{p} \approx 835 \mathrm{~J} / \mathrm{kg} \cdot \mathrm{K}$ is specific heat [25] and $\kappa=39$ $\mathrm{W} / \mathrm{m} \cdot \mathrm{K}$ is the thermal conductivity coefficient for iron in the molten state [25]. These parameters give the value of the thermal diffusivity $\chi=\kappa / \rho_{0} c_{p} \approx$ $6.7 \cdot 10^{-6} \mathrm{~m}^{2} / \mathrm{s}$, which turns out to be much less than the magnetic viscosity coefficient $\eta=1 / \mu \sigma \approx$ $2.65 \mathrm{~m}^{2} / \mathrm{s}: \eta \gg \chi$. The value $\mathrm{R}_{\mathrm{TM}} \approx 2$ was obtained for variations of the thermoelectromotive force coefficient $\Delta \alpha=3 \cdot 10^{-4} \mathrm{~V} / \mathrm{K}$ and temperature $\Delta T=2000 \mathrm{~K}$, and with the increase $\mathrm{R}_{\mathrm{TM}} \approx 20$ of the variations of the thermoelectromotive force coefficient to $\Delta \alpha \approx 10^{-3} \mathrm{~V} / \mathrm{K}$.

2. If the medium rotates nonuniformly $(\mathrm{Ro} \neq 0)$ but without an external magnetic field $\left(\mathbf{B}_{0}=0\right)$, then expression (15) takes the form

$$
\begin{gathered}
\operatorname{Ra}_{\mathrm{st}}=\frac{\left(k^{2}+\pi^{2}\right)^{3}}{k^{2}}+\frac{\pi^{2} \mathrm{Ta}}{k^{2}}(1+\mathrm{Ro}) \\
-\mathrm{R}_{\mathrm{TM}}\left(k^{2}+\pi^{2}+\frac{\pi^{2} \mathrm{Ta}}{\left(k^{2}+\pi^{2}\right)^{2}}(1+\mathrm{Ro})\right) .
\end{gathered}
$$

We also obtained the well-known result [26] for the case of a non-conductive $(\sigma=0)$ and uniformly rotating $(\mathrm{Ro}=0)$ medium from expression $(17)$ :

$$
\mathrm{Ra}_{\mathrm{st}}=\frac{\left(k^{2}+\pi^{2}\right)^{3}}{k^{2}}+\frac{\pi^{2} \mathrm{Ta}}{k^{2}}
$$

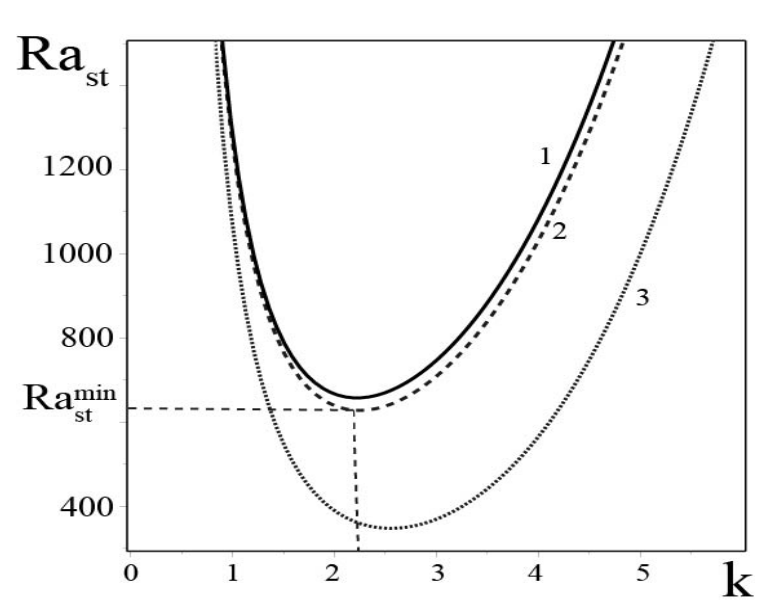

Fig. 2. Dependence of the stationary Rayleigh number $\mathrm{Ra}_{\mathrm{st}}$ on wavenumbers $k$ in the absence of rotation Ta $=0$ and magnetic field $\mathbf{B}_{0}=0$. Curve 1 corresponds to the parameter value $R_{T M}=0$, curve $2-R_{T M}=2$, curve $3-R_{T M}=20$.

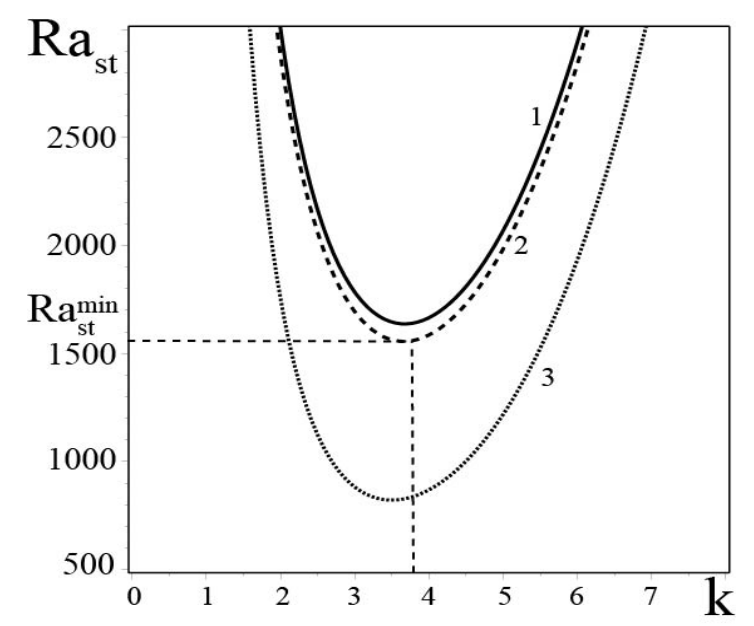

Fig. 3. Dependence of the stationary Rayleigh number Ra $\mathrm{ast}_{\mathrm{s}}$ on the wavenumbers $k$ for a medium uniformly rotating with the Taylor number $\mathrm{Ta}=946(\mathrm{Ro}=0)$ without an external magnetic field $\mathbf{B}_{0}=0$. Curve 1 corresponds to the parameter value $R_{\mathrm{TM}}=0$, curve $2-R_{\mathrm{TM}}=2$, curve $3-R_{\mathrm{TM}}=20$.

Similarly, we calculated the minimum value of the critical Rayleigh number $\mathrm{Ra}_{\mathrm{st}}^{\min }$ using formula (17) at $k=k_{c}$, which satisfies the following relation

$$
\begin{aligned}
& 2\left(\frac{k_{c}}{\pi}\right)^{6}+3\left(\frac{k_{c}}{\pi}\right)^{4}=1+\left(\frac{k_{c}^{4}}{\pi^{6}}\right) \mathrm{R}_{\mathrm{TM}} \\
& +\frac{\mathrm{Ta}}{\pi^{4}}(1+\mathrm{Ro})\left(1-\mathrm{R}_{\mathrm{TM}} \frac{k_{c}^{4}}{\left(k^{2}+\pi^{2}\right)^{3}}\right) .
\end{aligned}
$$

Fig. 3 shows the dependence of the critical (stationary) Rayleigh number $\mathrm{Ra}_{\mathrm{st}}$ (17) on wavenumbers $k$ in the presence $\mathrm{R}_{\mathrm{TM}} \neq 0$ and absence $\mathrm{R}_{\mathrm{TM}}=0$ 
of the influence of TM effects. Here we observe that with the increase of the coefficient $R_{T M}$ the minimum value of the critical Rayleigh number $\mathrm{Ra}_{\mathrm{st}}^{\mathrm{min}}$, for a uniformly rotating medium with the Taylor number $\mathrm{Ta}=946$, decreases, i.e. the threshold for the development of instability decreases. The Taylor number $\mathrm{Ta}=946$ was calculated for the parameters of the Earth's core: $\Omega_{0}=4 \cdot 10^{-5} \mathrm{~s}^{-1}$ is the angular velocity of rotation; $\nu=2.6 \mathrm{~m}^{2} / \mathrm{s}$ is the coefficient of hydrodynamic viscosity, which is considered equal to the coefficient of magnetic viscosity $\mathrm{Pm}=1 ; h=10^{3} \mathrm{~m}$ is the thickness of the convective layer. The estimates of the physical values of the Earth's core given in [25] have a fairly wide interval so we chose the above values of density $\rho_{0}$, electrical conductivity $\sigma$, thermal diffusivity $\chi$, viscosity $(\nu, \eta)$, temperature $T_{0}$, thermoelectromotive force coefficient $\alpha$, etc. convenient for numerical calculations and reasonable physical interpretation of the results.

Next, we fix the value of the coefficient $R_{T M}=2$, and the Rossby number Ro will be varied. Fig. 4 shows that with the increase of the positive profile of the Rossby number Ro the minimum value of the critical Rayleigh number also increases $\mathrm{Ra}_{\mathrm{st}}^{\mathrm{min}}$, i.e., the threshold for the development of instability increases. On the other hand, we observe the decrease of the critical Rayleigh number for negative rotation profiles $(\mathrm{Ro}=-1)$ (curve 3 ), i.e. the threshold for the development of instability is lower compared to the case of a uniform $(\mathrm{Ro}=0)$ (curve 2 ) and nonuniform $($ Ro $=2)$ (curve 1) rotation.

3. Let us consider the case when there is no rotation $(\mathrm{Ta}=0, \mathrm{Ro}=0)$ but there is an external magnetic field $\left(\mathbf{B}_{0} \neq 0\right)$. Then from expression (15) we found the critical value of the Rayleigh number:

$$
\begin{aligned}
\mathrm{Ra}_{\mathrm{st}} & =\frac{\left(k^{2}+\pi^{2}\right)^{3}}{k^{2}} \cdot\left(1+\frac{\pi^{2} \mathrm{Q}}{\left(k^{2}+\pi^{2}\right)^{2}}\right) \\
& -\mathrm{R}_{\mathrm{TM}}\left(k^{2}+\pi^{2}\right) .
\end{aligned}
$$

If $\mathrm{R}_{\mathrm{TM}}=0$, then we may obtain the result known from monograph [26]. The minimum value of the critical Rayleigh number $\mathrm{Ra}_{\mathrm{st}}^{\mathrm{min}}$ is determined from formula (18) at $k=k_{c}$, which satisfies the following relation

$2\left(\frac{k_{c}}{\pi}\right)^{6}+3\left(\frac{k_{c}}{\pi}\right)^{4}=1+\frac{\mathrm{Q}}{\pi^{2}}+\mathrm{R}_{\mathrm{TM}}\left(\frac{k_{c}^{4}}{\pi^{6}}\right)$

The graph in Fig. 5 shows the dependence of the critical (stationary) Rayleigh number $\mathrm{Ra}_{\text {st }}$ (18) on the wavenumbers $k$. The magnitude of the external poloidal (or meridional) magnetic field emerging from the core to the Earth's surface is of the order of $B_{0}=10^{-1} \mathrm{~T}$ [25], which will correspond to the Chandrasekhar number $\mathrm{Q}=1.68 \cdot 10^{5}$. In Fig. 5 we observe the decrease of the minimum critical Rayleigh number $\mathrm{Ra}_{\mathrm{st}}^{\mathrm{min}}$ with an increase of the coefficient $R_{\mathrm{TM}}$, which corresponds to a decrease of the threshold for the development of instability. Curve 1 is plotted for the case $R_{\mathrm{TM}}=0$, curve 2 $-\mathrm{R}_{\mathrm{TM}}=2$, curve $3-\mathrm{R}_{\mathrm{TM}}=20$.

Thus, the conclusions about the lowering of the threshold of convective instability taking into account TM effects remain valid even in the presence of an external magnetic field.

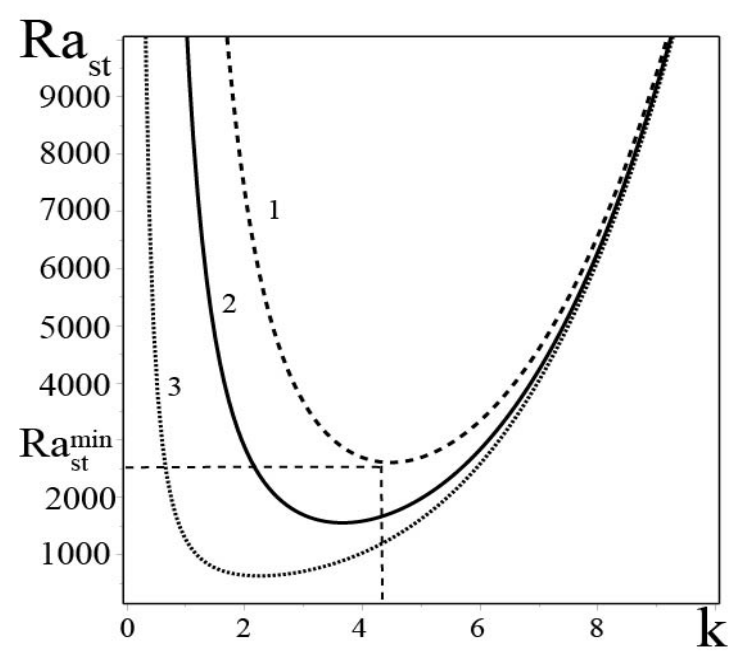

Fig. 4. Dependence of the stationary Rayleigh number $\mathrm{Ra}_{\mathrm{st}}$ on wavenumbers $k$ for an inhomogeneously rotating medium with the Taylor number $\mathrm{Ta}=946$ without an external magnetic field $\mathbf{B}_{0}=0$ at the constant parameter $\mathrm{R}_{\mathrm{TM}}=2$. Curves 1,2,3 correspond to Rossby numbers $\operatorname{Ro}=2$, Ro $=0$, Ro $=-1$, respectively.

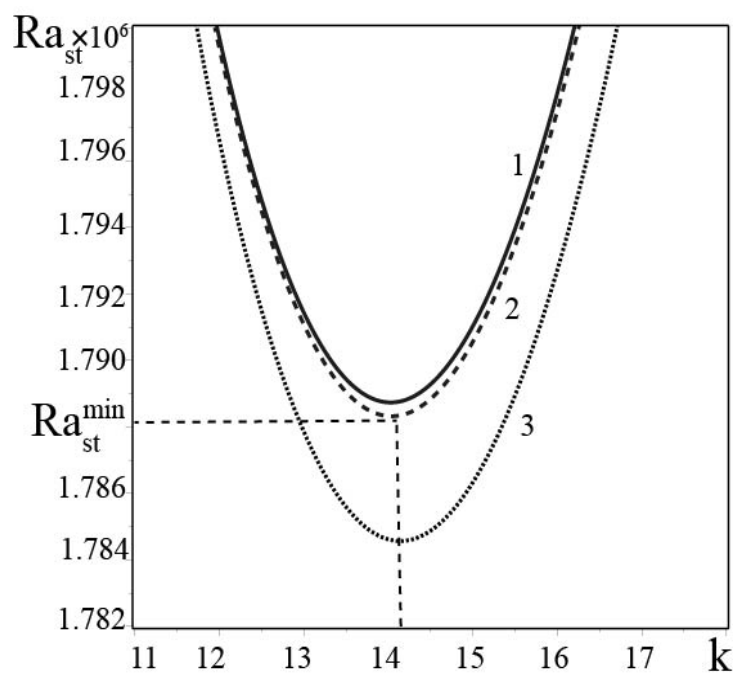

Fig. 5. Dependence of the stationary Rayleigh number $\mathrm{Ra}_{\text {st }}$ on wavenumbers $k$ for a non-rotating medium $(\mathrm{Ta}=0)$ in an external magnetic field $B_{0}=10^{-1} \mathrm{~T}$ at different values of the parameter $R_{\mathrm{TM}}$. Curves $1,2,3$ correspond to $\mathrm{R}_{\mathrm{TM}}=0$, $\mathrm{R}_{\mathrm{TM}}=2, \mathrm{R}_{\mathrm{TM}}=20$, respectively.

All the limiting cases considered above are completely in agreement with the conclusions of Chandrasekhar's works [26] on the suppression of convection by the effects of rotation and an external magnetic field. 


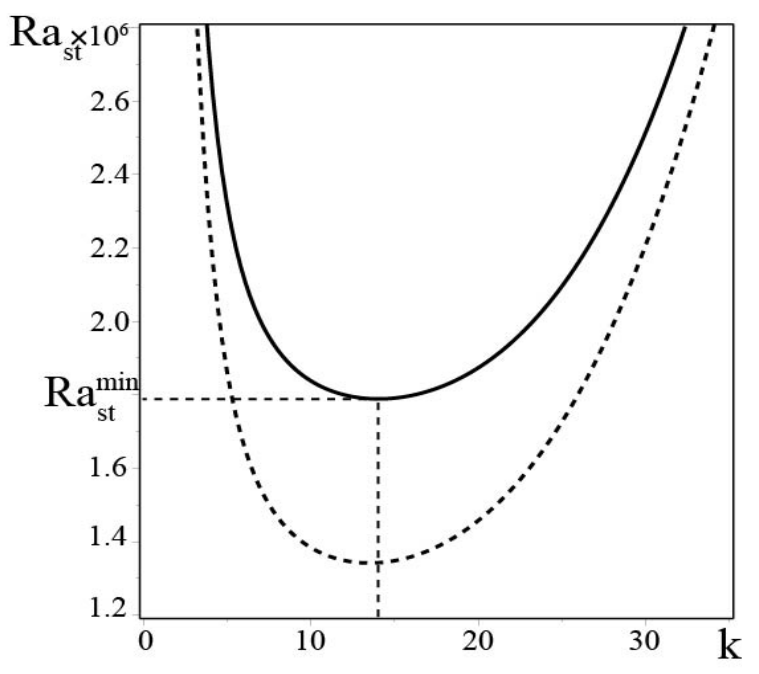

Fig. 6. Dependence of the stationary Rayleigh number $\mathrm{Ra}_{\mathrm{st}}$ on wavenumbers $k$ for a rotating medium $(\mathrm{Ta}=946, \mathrm{Ro}=-1$ ) in an external magnetic field $B_{0}=10^{-1} \mathrm{~T}$ and the magnetic Prandtl number $\mathrm{Pm}=1$. The solid line corresponds to the case without taking into account TM effects $q_{\alpha}=0, R_{\alpha}=0$, and the dashed line for $q_{\alpha} \neq 0, R_{\alpha} \neq 0$.

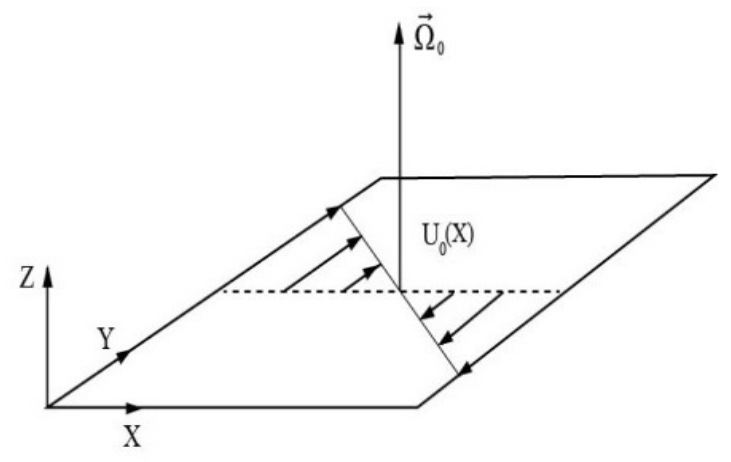

a)
Let us now proceed to study the general case of nonuniformly rotating stationary magnetoconvection taking into account TM effects. As before, we have calculated the convection parameters $\left(\mathrm{Q}, \mathrm{Ta}, \mathrm{Pm}, q_{\alpha}, R_{\alpha}\right)$ using the values of physical quantities $\left(\rho_{0}, \nu, \eta, \chi, T_{0}, \alpha, B_{0}, \Omega_{0}\right)$ for the Earth's core [25]: $\mathrm{Q}=1.68 \cdot 10^{5}, \mathrm{Ta}=946, \mathrm{Pm}=1$, $q_{\alpha} \operatorname{PmPr}^{-1} \approx 5.24 \cdot 10^{-8}, \mathrm{R}_{\alpha} \widetilde{\mathrm{Q}} \approx 2.5 \cdot 10^{9}$. Fig. 6 shows the minimum value of the critical Rayleigh number $\mathrm{Ra}_{\mathrm{st}}^{\min } 15$ for the case when there are no TM effects $\Delta \alpha=0$ (the medium is homogeneous in chemical composition). As seen from Fig. 6, when TM effects are taken into account the minimum critical Rayleigh number $\mathrm{Ra}_{\mathrm{st}}^{\mathrm{min}}$ decreases, i.e. the threshold for the onset of convective instability decreases. The dependence plot $\operatorname{Ra}_{\mathrm{st}}(k)$ (Fig. 6) is built for the Rayleigh rotation profile $(\mathrm{Ro}=-1)$. The dependence plot $\mathrm{Ra}_{\mathrm{st}}(k)$ for the profile of uniform rotation $(\mathrm{Ro}=0)$ and positive profile $(\mathrm{Ro}=2)$ has the similar view. It follows from the results obtained above that the generation of a magnetic field using TM effects promotes the development of convective instability. Magnetic and thermal perturbations are localized in convective cells on scales of the order $l \sim k_{c}^{-1}$.

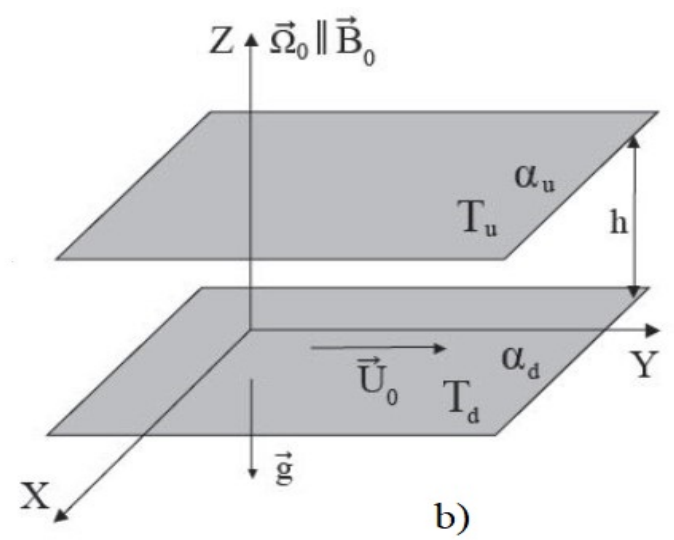

Fig. 7. a) Diagram of a shear flow in rotating flows. In the local Cartesian system, this flow is approximated as a linear shear with velocity $\mathbf{U}_{0}(X)$; b) Cartesian approximation of the problem for a nonuniformly rotating magnetic convection. Nonuniform rotation in the local Cartesian system of coordinates consists of rotation with constant angular velocity $\Omega_{0}$ and shear velocity $\mathbf{U}_{0}$ directed along the Y-axis.

\section{WEAKLY NONLINEAR REGIME OF CONVECTION TAKING INTO ACCOUNT THERMOMAGNETIC EFFECTS}

In this section, we will consider the weakly nonlinear convection regime, limiting ourselves, as in the previous section, to TM effects associated with the inhomogeneity of the thermoelectromotive force coefficient and " magnetization" of the heat flux (Leduc-Righi effect). By weakly nonlinear convection we mean the interaction between small amplitudes of convective cells, which can be described as follows. Let the small amplitude of convective cells be of order $O\left(\epsilon^{1}\right)$, then the interaction of the cells with each other leads to the second harmonic and nonlinearity of the order $O\left(\epsilon^{2}\right)$, and then to nonlinearity $O\left(\epsilon^{3}\right)$, etc. In this case, the nonlinear terms in equations (9) are considered as perturbed response for the linear convection problem. In this case, the Rayleigh parameter Ra controlling convection is close to critical $\mathrm{Ra}_{c}$. Since the influence of unstable modes is small, our task is to obtain an equation that describes the interaction between these modes.

\section{A. Equations of nonlinear convection in rotating flows of a magnetized fluid with a shear velocity}

To describe the nonlinear convective phenomena in the nonuniformly rotating layer of the electrically conducting fluid, it is convenient to turn from the cylindrical coordinate system $(R, \varphi, z)$ to the local Cartesian $(X, Y, Z)$ one. If we consider a fixed region of the fluid layer with a radius $R_{0}$ and angular velocity of rotation $\Omega_{0}=$ $\Omega\left(R_{0}\right)$, then the coordinates $X=R-R_{0}$ correspond 
to the radial direction, $Y=R_{0}\left(\varphi-\varphi_{0}\right)$ to azimuthal, and $Z=z$ to vertical (see Fig. 7 ). In this case, the fluid layer inhomogeneous rotation can be represented locally as the rotation with the constant angular velocity $\Omega_{0}$ and azimuthal shear [28] whose velocity profile is locally linear $\mathbf{U}_{0}=-q \Omega_{0} X \mathbf{e}_{Y}$, where $q \equiv-d \ln \Omega / d \ln R$ is the dimensionless shear parameter determined from the profile of the angular velocity of rotation $\Omega(R)=$ $\Omega_{0}\left(R / R_{0}\right)^{-q}$. The shear parameter $q$ is bound up with the hydrodynamic Rossby number Ro $=\frac{R}{2 \Omega} \frac{\partial \Omega}{\partial R}$ by the relation $q=-2$ Ro. Note that the accretion disk with a shear parameter $q=3 / 2$, Ro $=-3 / 4$ corresponds to the Keplerian disk; $q=2$, Ro $=-1$ corresponds to the disk with a constant angular momentum, or the Rayleigh rotation profile. The case of $q=1, \mathrm{Ro}=-1 / 2$ corresponds to the system with a flat rotation curve, and that of $q=0$, Ro $=0$ to the homogeneous (or solid-state) rotation with a constant angular velocity. As before, we assume that the direction of the external magnetic field $\mathbf{B}_{0}$ coincides with the axis of rotation of the fluid $\mathbf{\Omega} \| O Z$.

The equations for the perturbations $(\mathbf{u}=$ $\left.\left(u_{X}, u_{Y}, u_{Z}\right), \quad \mathbf{b}=\left(b_{X}, b_{Y}, b_{Z}\right), p, \theta\right)$ in the local Cartesian coordinate system take the following form:

$$
\begin{gathered}
\left(\frac{\partial}{\partial t}-\nu \nabla^{2}\right) u_{X}+(\mathbf{u} \cdot \nabla) u_{X}-2 \Omega_{0} u_{Y}=-\frac{1}{\rho_{0}} \frac{\partial \widetilde{p}}{\partial X}+\frac{1}{\mu \rho_{0}}(\mathbf{b} \cdot \nabla) b_{X}+\frac{B_{0}}{\mu \rho_{0}} \frac{\partial b_{X}}{\partial Z} \\
\left(\frac{\partial}{\partial t}-\nu \nabla^{2}\right) u_{Y}+(\mathbf{u} \cdot \nabla) u_{Y}+2 \Omega_{0} u_{X}(1+\operatorname{Ro})=\frac{1}{\mu \rho_{0}}(\mathbf{b} \cdot \nabla) b_{Y}+\frac{B_{0}}{\mu \rho_{0}} \frac{\partial b_{Y}}{\partial Z} \\
\left(\frac{\partial}{\partial t}-\nu \nabla^{2}\right) u_{Z}+(\mathbf{u} \cdot \nabla) u_{Z}=-\frac{1}{\rho_{0}} \frac{\partial \widetilde{p}}{\partial Z}+g \beta_{T} \theta+\frac{1}{\mu \rho_{0}}(\mathbf{b} \cdot \nabla) b_{Z}+\frac{B_{0}}{\mu \rho_{0}} \frac{\partial b_{Z}}{\partial Z} \\
\left(\frac{\partial}{\partial t}-\eta \nabla^{2}\right) b_{X}-B_{0} \frac{\partial u_{X}}{\partial Z}+(\mathbf{u} \cdot \nabla) b_{X}-(\mathbf{b} \cdot \nabla) u_{X}=0 \\
\left(\frac{\partial}{\partial t}-\eta \nabla^{2}\right) b_{Y}-B_{0} \frac{\partial u_{Y}}{\partial Z}-2 \Omega_{0} \operatorname{Ro} b_{X}+(\mathbf{u} \cdot \nabla) b_{Y}-(\mathbf{b} \cdot \nabla) u_{Y}=\frac{\Delta \alpha}{h} \frac{\partial \theta}{\partial X} \\
\left(\frac{\partial}{\partial t}-\eta \nabla^{2}\right) b_{Z}-B_{0} \frac{\partial u_{Z}}{\partial Z}+(\mathbf{u} \cdot \nabla) b_{Z}-(\mathbf{b} \cdot \nabla) u_{Z}=0, \\
-\frac{1}{\rho_{0} c_{p} \mu}\left(-\alpha \frac{\partial \theta}{\partial X} \frac{\partial b_{Y}}{\partial Z}+\alpha \frac{\partial \theta}{\partial Z} \frac{\partial b_{Y}}{\partial X}+\theta \frac{\Delta \alpha}{h} \frac{\partial b_{Y}}{\partial X}\right)-\chi_{\wedge}\left(\frac{\partial b_{Y}}{\partial X} \frac{\partial \theta}{\partial Z}-\frac{\partial b_{Y}}{\partial Z} \frac{\partial \theta}{\partial X}\right) \\
\left(\frac{\partial}{\partial t}-\chi \nabla^{2}\right) \theta-u_{Z} \cdot \frac{\Delta T}{h}+(\mathbf{u} \cdot \nabla) \theta=-\frac{\alpha T_{0}}{\rho_{0} c_{p} \mu h}\left(\frac{\Delta \alpha}{\alpha}+\frac{\Delta T}{T_{0}}\right) \frac{\partial b_{Y}}{\partial X}-\chi_{\wedge} \frac{\Delta T}{h} \frac{\partial b_{Y}}{\partial X}
\end{gathered}
$$

where the pressure $\widetilde{p}$ also includes the perturbed magnetic pressure $p_{m}=\frac{1}{2 \mu}\left(2 \mathbf{B}_{0} \cdot \mathbf{b}+\mathbf{b}^{2}\right): \widetilde{p}=p+p_{m}$. In equations (19)-(25), we assumed that all perturbed quantities depend only on two variables $(X, Z)$, i.e., we consider the dynamics of axisymmetric perturbations. The solenoidality equations for axisymmetric velocity and magnetic field perturbations take the form

$$
\frac{\partial u_{X}}{\partial X}+\frac{\partial u_{Z}}{\partial Z}=0, \quad \frac{\partial b_{X}}{\partial X}+\frac{\partial b_{Z}}{\partial Z}=0
$$

Considering equations (26), we can introduce two scalar functions: hydrodynamic stream function $\psi$ and magnetic $\phi$, for which the following relations hold:

$$
u_{X}=-\frac{\partial \psi}{\partial Z}, \quad u_{Z}=\frac{\partial \psi}{\partial X}, \quad b_{X}=-\frac{\partial \phi}{\partial Z}, \quad b_{Z}=\frac{\partial \phi}{\partial X} .
$$

It is convenient to transform Eqs. (19)-(25) to dimensionless variables using terms of stream function $\psi$ and function $\phi$

$$
(X, Z)=h\left(x^{*}, z^{*}\right), t=\frac{h^{2}}{\nu} t^{*}, \psi=\chi \psi^{*}, \phi=h B_{0} \phi^{*}
$$




$$
u_{Y}=\frac{\chi}{h} v^{*}, b_{Y}=B_{0} \widetilde{v}^{*}, \theta=(\Delta T) \theta^{*} .
$$

Omitting the asterisk, we rewrite these equations in dimensionless variables:

$$
\begin{gathered}
\left(\frac{\partial}{\partial t}-\nabla^{2}\right) \nabla^{2} \psi+\sqrt{\operatorname{Ta}} \frac{\partial v}{\partial z}-\operatorname{Pr}^{-1} \operatorname{Q} \frac{\partial}{\partial z} \nabla^{2} \phi-\operatorname{Ra} \frac{\partial \theta}{\partial x}=\operatorname{Pr}^{-1} \mathrm{Q} \cdot J\left(\phi, \nabla^{2} \phi\right)-\operatorname{Pr}^{-1} \cdot J\left(\psi, \nabla^{2} \psi\right), \\
\left(\frac{\partial}{\partial t}-\nabla^{2}\right) v-\sqrt{\operatorname{Ta}}(1+\operatorname{Ro}) \frac{\partial \psi}{\partial z}-\operatorname{Pr}^{-1} \mathrm{Q} \frac{\partial \widetilde{v}}{\partial z}=\operatorname{Pr}^{-1} \mathrm{P} \cdot J(\phi, \widetilde{v})-\operatorname{Pr}^{-1} \cdot J(\psi, v), \\
\left(\frac{\partial}{\partial t}-\operatorname{Pm}^{-1} \nabla^{2}\right) \phi-\operatorname{Pr}^{-1} \frac{\partial \psi}{\partial z}=-\operatorname{Pr}^{-1} J(\psi, \phi), \\
\left(\frac{\partial}{\partial t}-\operatorname{Pm}^{-1} \nabla^{2}\right) \widetilde{v}-\operatorname{Pr}^{-1} \frac{\partial v}{\partial z}+\operatorname{Ro} \sqrt{\operatorname{Ta}} \frac{\partial \phi}{\partial z}-\operatorname{Pm}^{-1} R_{\alpha} \frac{\partial \theta}{\partial x}=\operatorname{Pr}^{-1}(J(\phi, v)-J(\psi, \widetilde{v})), \\
\left(\operatorname{Pr} \frac{\partial}{\partial t}-\nabla^{2}\right) \theta-\frac{\partial \psi}{\partial x}+q_{\alpha} \frac{\partial \widetilde{v}}{\partial x}=-J(\psi, \theta)-q_{\alpha}^{(1)} \theta \cdot \frac{\partial \widetilde{v}}{\partial x}+q_{\alpha}^{(2)} J(\theta, \widetilde{v}),
\end{gathered}
$$

where new designations for dimensionless parameters are introduced

$$
q_{\alpha}^{(1)}=\frac{\Delta \alpha B_{0}}{\rho_{0} c_{p} \mu \chi}, \quad q_{\alpha}^{(2)}=\frac{\alpha B_{0}}{\rho_{0} c_{p} \mu \chi}\left(\frac{\mu \mathcal{L}}{\alpha}+1\right) .
$$

This system of equations is supplemented with the following boundary conditions:

$$
\begin{gathered}
\left.\psi\right|_{z=0, h}=\left.\nabla^{2} \psi\right|_{z=0, h}=\left.\frac{d v}{d z}\right|_{z=0, h}=\left.\widetilde{v}\right|_{z=0, h}=0, \\
\left.\frac{d \phi}{d z}\right|_{z=0, h}=\left.\theta\right|_{z=0, h}=0 .
\end{gathered}
$$

In the absence of thermal and thermomagnetic phenomena, the system of equations (27) was used to study the saturation mechanism of the standard MRI [29]. In the case when there are no TM effects, the system of equations (27) was used to study weakly nonlinear and chaotic convection regimes in a nonuniformly rotating plasma in an axial magnetic field [23].

\section{B. Equation of finite amplitude for stationary convection}

We will obtain an equation for the finite amplitude of the magnetic field generated by the RayleighBenard convection and thermomagnetic instability in a nonuniformly rotating electrically conductive fluid in an external uniform magnetic field using the weakly nonlinear theory (see for example [30]). We represent all the variables in equations (27) in the form of an asymptotic expansion:

$$
\begin{aligned}
& \mathrm{Ra}=\mathrm{Ra}_{c}+\epsilon^{2} \mathrm{Ra}_{2}+\epsilon^{4} \mathrm{Ra}_{4}+\ldots, \\
& \psi=\epsilon \psi_{1}+\epsilon^{2} \psi_{2}+\epsilon^{3} \psi_{3}+\ldots, \\
& v=\epsilon v_{1}+\epsilon^{2} v_{2}+\epsilon^{3} v_{3}+\ldots, \\
& \phi=\epsilon \phi_{1}+\epsilon^{2} \phi_{2}+\epsilon^{3} \phi_{3}+\ldots, \\
& \widetilde{v}=\epsilon \widetilde{v}_{1}+\epsilon^{2} \widetilde{v}_{2}+\epsilon^{3} \widetilde{v}_{3}+\ldots \\
& \theta=\varepsilon \theta_{1}+\epsilon^{2} \theta_{2}+\epsilon^{3} \theta_{3}+\ldots
\end{aligned}
$$

where $\epsilon$ is the small parameter of the expansion, which is the relative deviation of the Rayleigh number Ra from the critical value $\mathrm{Ra}_{c}$ :

$$
\epsilon^{2}=\frac{\mathrm{Ra}-\mathrm{Ra}_{c}}{\mathrm{Ra}_{c}} \ll 1 .
$$

We assume that the amplitudes of the perturbed quantities depend only on the slow time $\tau=\epsilon^{2} t$. Substituting expansions (29) into the system of equations (27), we solve it for different orders in $\epsilon$. For simplicity, we will take into account the nonlinear terms in (27) only in the heat balance equation.

In the first order in $\epsilon$, we obtain the equation

$$
\widehat{L} M_{1}=0,
$$

where

$$
M_{1}=\left(\begin{array}{c}
\psi_{1} \\
v_{1} \\
\phi_{1} \\
\widetilde{v}_{1} \\
\theta_{1}
\end{array}\right) \text {, }
$$


$\widehat{L}$ is the matrix operator of the form:

$$
\widehat{L}=\left(\begin{array}{ccccc}
-\nabla^{4} & \sqrt{\mathrm{Ta}} \frac{\partial}{\partial z} & -\widetilde{\mathrm{Q}} \frac{\partial}{\partial z} \nabla^{2} & 0 & -\operatorname{Ra}_{c} \frac{\partial}{\partial x} \\
-\sqrt{\operatorname{Ta}}(1+\operatorname{Ro}) \frac{\partial}{\partial z} & -\nabla^{2} & 0 & -\widetilde{\mathrm{Q}} \frac{\partial}{\partial z} & 0 \\
-\operatorname{Pr}^{-1} \frac{\partial}{\partial z} & 0 & -\operatorname{Pm}^{-1} \nabla^{2} & 0 & 0 \\
0 & -\operatorname{Pr}^{-1} \frac{\partial}{\partial z} & \operatorname{Ro} \sqrt{\mathrm{Ta}} \frac{\partial}{\partial z} & -\operatorname{Pm}^{-1} \nabla^{2} & -\operatorname{Pm}^{-1} R_{\alpha} \frac{\partial}{\partial x} \\
-\frac{\partial}{\partial x} & 0 & 0 & q_{\alpha} \frac{\partial}{\partial x} & -\nabla^{2}
\end{array}\right) .
$$

The solutions of the system of equations (30) with the boundary conditions of (IV A) have, respectively, the form:

$$
\begin{gathered}
\psi_{1}=A(\tau) \sin k_{c} x \sin \pi z, \quad \phi_{1}=\frac{A(\tau) \pi \mathrm{Pm}}{a^{2} \mathrm{Pr}} \sin k_{c} x \cos \pi z, \\
\theta_{1}=\frac{A(\tau) k_{c}}{a^{2}}\left(1+q_{\alpha} \cdot \Pi_{\alpha}\right) \cos k_{c} x \sin \pi z, \quad \widetilde{v}_{1}=-\Pi_{\alpha} A(\tau) \sin k_{c} x \sin \pi z, \\
v_{1}=\frac{\pi \sqrt{\mathrm{Ta}}\left[(1+\mathrm{Ro})\left(a^{4}-k_{c}^{2} q_{\alpha} R_{\alpha}\right)+\pi^{2} \mathrm{QP} \mathrm{mRo}\right]-\pi k_{c}^{2} \widetilde{\mathrm{Q}} R_{\alpha}}{a^{2}\left(a^{4}+\pi^{2} \mathrm{Q}-k_{c}^{2} q_{\alpha} R_{\alpha}\right)} A(\tau) \sin k_{c} x \cos \pi z,
\end{gathered}
$$

where

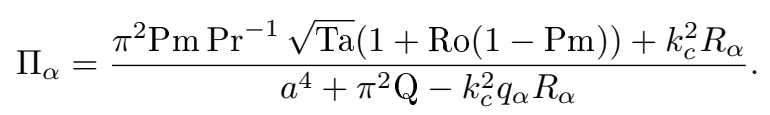

The critical value of the Rayleigh number $\mathrm{Ra}_{c}$ for stationary convection is found from the first equation of system (30) and has the form of the formula (15) obtained in the linear theory. The amplitude $A(\tau)$ is still unknown.

For the second-order in $\epsilon$, we have the following equation:

$$
\widehat{L} M_{2}=N_{2},
$$

where $M_{2}=\left(\begin{array}{c}\psi_{2} \\ v_{2} \\ \phi_{2} \\ \widetilde{v}_{2} \\ \theta_{2}\end{array}\right), \quad N_{2}=\left(\begin{array}{c}N_{21} \\ N_{22} \\ N_{23} \\ N_{24} \\ N_{25}\end{array}\right)$,

$$
\begin{gathered}
N_{21}=N_{22}=N_{23}=N_{24}=0 \\
N_{25}=-\left(\frac{\partial \psi_{1}}{\partial x} \frac{\partial \theta_{1}}{\partial z}-\frac{\partial \theta_{1}}{\partial x} \frac{\partial \psi_{1}}{\partial z}\right)-q_{\alpha}^{(1)} \theta_{1} \frac{\partial \widetilde{v}_{1}}{\partial x}+q_{\alpha}^{(2)}\left(\frac{\partial \theta_{1}}{\partial x} \frac{\partial \widetilde{v}_{1}}{\partial z}-\frac{\partial \theta_{1}}{\partial z} \frac{\partial \widetilde{v}_{1}}{\partial x}\right) .
\end{gathered}
$$

Using solutions (31) and boundary conditions (IV A), we can find solutions of equations (32):

$$
\begin{gathered}
\psi_{2}=0, \quad \phi_{2}=0, \quad v_{2}=0, \quad \widetilde{v}_{2}=0, \\
\theta_{2}=-\frac{A^{2}(\tau) k_{c}^{2}}{8 \pi a^{2}}\left(1-q_{\alpha}^{(2)} \cdot \Pi_{\alpha}\right)\left(1+q_{\alpha} \cdot \Pi_{\alpha}\right) \sin (2 \pi z) .
\end{gathered}
$$

To analyze the intensity of the heat transfer, a horizontally-averaged heat flux is introduced at the boundary of the layer of the electrically conducting fluid (Nusselt number)

$$
\mathrm{Nu}(\tau)=1+\frac{\left[\frac{k_{c}}{2 \pi} \int_{0}^{2 \pi / k_{c}}\left(\frac{\partial \theta_{2}}{\partial z}\right) d x\right]_{z=0}}{\left[\frac{k_{c}}{2 \pi} \int_{0}^{2 \pi / k_{c}}\left(\frac{\partial T_{0}}{\partial z}\right) d x\right]_{z=0}}=1+\frac{k_{c}^{2}}{4 a^{2}} A^{2}(\tau) .
$$


The heat flow intensity (of Nusselt number $\mathrm{Nu}$ ) will be analyzed after the expression for the amplitude $A(\tau)$ is obtained.

For the third order of $\epsilon$ we can find:

$$
\widehat{L} M_{3}=N_{3},
$$

where $M_{3}=\left(\begin{array}{c}\psi_{3} \\ v_{3} \\ \phi_{3} \\ \widetilde{v}_{3} \\ \theta_{3}\end{array}\right), \quad N_{3}=\left(\begin{array}{c}N_{31} \\ N_{32} \\ N_{33} \\ N_{34} \\ N_{35}\end{array}\right)$;

$$
\begin{gathered}
N_{31}=-\frac{\partial}{\partial \tau} \nabla^{2} \psi_{1}+\operatorname{Ra}_{2} \frac{\partial \theta_{1}}{\partial x}=\left(a^{2} \frac{\partial A(\tau)}{\partial \tau}-\operatorname{Ra}_{2} \frac{k_{c}^{2} A(\tau)}{a^{2}}\left(1+q_{\alpha} \cdot \Pi_{\alpha}\right)\right) \sin k_{c} x \sin \pi z \\
N_{32}=-\frac{\partial v_{1}}{\partial \tau}=-\frac{\pi \sqrt{\mathrm{Ta}}\left[(1+\mathrm{Ro})\left(a^{4}-k_{c}^{2} q_{\alpha} R_{\alpha}\right)+\pi^{2} \mathrm{QPmRo}\right]-\pi k_{c}^{2} \widetilde{\mathrm{Q}} R_{\alpha}}{a^{2}\left(a^{4}+\pi^{2} \mathrm{Q}-k_{c}^{2} q_{\alpha} R_{\alpha}\right)} \frac{\partial A(\tau)}{\partial \tau} \sin k_{c} x \cos \pi z \\
N_{33}=-\frac{\partial \phi_{1}}{\partial \tau}=-\frac{\pi \mathrm{Pm}}{a^{2} \operatorname{Pr}} \cdot \frac{\partial A(\tau)}{\partial \tau} \sin k_{c} x \cos \pi z \\
N_{34}=-\frac{\partial \widetilde{v}_{1}}{\partial \tau}=\Pi_{\alpha} \cdot \frac{\partial A(\tau)}{\partial \tau} \sin k_{c} x \sin \pi z \\
N_{35}=-\operatorname{Pr} \frac{\partial \theta_{1}}{\partial \tau}-\frac{\partial \psi_{1}}{\partial x} \frac{\partial \theta_{2}}{\partial z}-q_{\alpha}^{(1)} \theta_{2} \frac{\partial \widetilde{v}_{1}}{\partial x}-q_{\alpha}^{(2)} \frac{\partial \theta_{2}}{\partial z} \frac{\partial \widetilde{v}_{1}}{\partial x}=-\operatorname{Pr} \frac{k_{c}}{a^{2}} \frac{\partial A(\tau)}{\partial \tau} \cos k_{c} x \sin \pi z \\
+\frac{k_{c}^{3} A^{3}(\tau)}{4 a^{2}}\left(1-q_{\alpha}^{(2)} \cdot \Pi_{\alpha}\right)^{2}\left(1+q_{\alpha} \cdot \Pi_{\alpha}\right) \cos k_{c} x \sin \pi z \cos 2 \pi z \\
-q_{\alpha}^{(1)} \cdot \frac{k_{c}^{3} A^{3}(\tau)}{8 \pi a^{2}}\left(1-q_{\alpha}^{(2)} \cdot \Pi_{\alpha}\right)\left(1+q_{\alpha} \cdot \Pi_{\alpha}\right) \Pi_{\alpha} \cos k_{c} x \sin \pi z \sin 2 \pi z
\end{gathered}
$$

The solvability condition for the chain of nonlinear equations (32), (35) is known as Fredholm's alternative (see, for example, [31])

$$
\left\langle M_{1}^{\dagger}, R . H .\right\rangle=0
$$

where R.H. are the right sides of the perturbed equations with nonlinear terms. The matrix $M_{1}^{\dagger}=\left(\psi_{1}^{\dagger}, \theta_{1}^{\dagger}, \phi_{1}^{\dagger}, v_{1}^{\dagger}\right)^{T r}$ is a nontrivial solution to the linear self-adjoint problem $\widehat{L}^{\dagger} M_{1}^{\dagger}=0$, where $\widehat{L}^{\dagger}$ is a self-adjoint operator, which is determined from the following relation

$$
\left\langle M_{1}^{\dagger}, \widehat{L} M_{1}\right\rangle \equiv\left\langle\widehat{L}^{\dagger} M_{1}^{\dagger}, M_{1}\right\rangle
$$

where $\langle$,$\rangle is the inner product, which here has the following definition:$

$$
\langle\mathbf{f}, \mathbf{g}\rangle=\int_{z=0}^{1} \int_{x=0}^{2 \pi / k_{c}} \mathbf{f} \cdot \mathbf{g} d x d z
$$

Using expression (36), we write the Fredholm solvability condition for third-order $O\left(\varepsilon^{3}\right)$ equations $(35)$ in the following form:

$$
\int_{z=0}^{1} \int_{x=0}^{2 \pi / k_{c}}\left[\widehat{\mathcal{K}} \widehat{\mathcal{M}} \psi_{1}^{\dagger} \cdot \mathcal{R}_{31}-\operatorname{Ra}_{c} \frac{\partial}{\partial x} \widehat{\mathcal{K}} \theta_{1}^{\dagger} \cdot \mathcal{R}_{32}+\widetilde{\mathrm{Q}} \nabla^{2} \widehat{\mathcal{K}} \widehat{\mathcal{M}} \phi_{1}^{\dagger} \cdot \mathcal{R}_{33}++\sqrt{\mathrm{Ta}} \frac{\partial}{\partial z} \widehat{\mathcal{M}} v_{1}^{\dagger} \cdot \mathcal{R}_{34}\right] d x d z=0
$$


where the notations are introduced

$$
\begin{aligned}
& \widehat{\mathcal{K}}=\sqrt{\mathrm{Ta}} \widehat{P} \frac{\partial}{\partial z}\left(\nabla^{4}+q_{\alpha} R_{\alpha} \frac{\partial^{2}}{\partial x^{2}}\right)+\widetilde{\mathrm{Q}} R_{\alpha} \frac{\partial^{3} \widehat{q}}{\partial x^{2} \partial z}, \\
& \widehat{\mathcal{M}}=-\frac{\partial^{2} \widehat{q}}{\partial x \partial z}\left(\nabla^{4}-\mathrm{Q} \frac{\partial^{2}}{\partial z^{2}}\right)+q_{\alpha} \operatorname{PmPr}^{-1} \sqrt{\mathrm{Ta}} \widehat{P} \frac{\partial^{4}}{\partial x \partial z^{3}}, \\
& \widehat{P}=(1+\operatorname{Ro}) \nabla^{4}-\operatorname{QPmRo} \frac{\partial^{2}}{\partial z^{2}}, \widehat{q}=\nabla^{4}+q_{\alpha} \operatorname{Pm}^{2} \operatorname{Pr}^{-1} \operatorname{Ro} \sqrt{\operatorname{Ta}} \frac{\partial^{2}}{\partial z^{2}}, \\
& \mathcal{R}_{31}=N_{31}, \quad \mathcal{R}_{32}=\left(\nabla^{4}-\mathrm{Q} \frac{\partial^{2}}{\partial z^{2}}\right) \mathrm{R}_{32}+q_{\alpha} \operatorname{PmPr}^{-1} \frac{\partial^{3} \mathrm{R}_{34}}{\partial x \partial z^{2}}, \\
& \mathcal{R}_{33}=N_{33} \operatorname{Pr}, \quad \mathcal{R}_{34}=\left(\nabla^{4}+q_{\alpha} R_{\alpha} \frac{\partial^{2}}{\partial x^{2}}\right) \mathrm{R}_{34}-\widetilde{\mathrm{Q}} R_{\alpha} \frac{\partial \mathrm{R}_{32}}{\partial x}, \\
& \mathrm{R}_{32}=\frac{\partial \nabla^{2}}{\partial z}\left(\nabla^{2} N_{35}+q_{\alpha} \operatorname{Pm} \frac{\partial N_{34}}{\partial x}\right)+q_{\alpha} \operatorname{Pm}^{2} \operatorname{Pr}^{-1} \operatorname{Ro} \sqrt{\mathrm{Ta}} \frac{\partial^{3} N_{33}}{\partial x \partial z^{2}}, \\
& \mathrm{R}_{34}=-\nabla^{4} N_{32}+\operatorname{QPr} \frac{\partial}{\partial z} \nabla^{2} N_{34}+\sqrt{\operatorname{Ta}} \operatorname{PrmPrRo} \frac{\partial^{2} N_{33}}{\partial z^{2}} .
\end{aligned}
$$

Expressions for $\psi_{1}^{\dagger}, \theta_{1}^{\dagger}, \phi_{1}^{\dagger}, v_{1}^{\dagger}$ are determined from the solution of the linear self-adjoint problem $\widehat{L}^{\dagger} M_{1}^{\dagger}=0$ :

$$
\begin{gathered}
\psi_{1}^{\dagger}=A(\tau) \sin k_{c} x \sin \pi z \\
\theta_{1}^{\dagger}=-\frac{A(\tau) k_{c}}{a^{2}}\left(1+q_{\alpha} \cdot \Pi_{\alpha}\right) \cos k_{c} x \sin \pi z \\
\phi_{1}^{\dagger}=-\frac{A(\tau) \pi \mathrm{Pm}}{a^{2} \mathrm{Pr}} \sin k_{c} x \cos \pi z \\
v_{1}^{\dagger}=-\frac{\pi \sqrt{\mathrm{Ta}}\left[(1+\mathrm{Ro})\left(a^{4}-k_{c}^{2} q_{\alpha} R_{\alpha}\right)+\pi^{2} \mathrm{QP} \mathrm{mRo}\right]-\pi k_{c}^{2} \widetilde{\mathrm{Q}} R_{\alpha}}{a^{2}\left(a^{4}+\pi^{2} \mathrm{Q}-k_{c}^{2} q_{\alpha} R_{\alpha}\right)} \cdot A(\tau) \sin k_{c} x \cos \pi z
\end{gathered}
$$

The self-adjoint matrix operator $\widehat{L}^{\dagger}$ takes the following form:

$$
\widehat{L}^{\dagger}=\left(\begin{array}{cccc}
\nabla^{4} \widehat{\mathcal{K}} \widehat{\mathcal{M}} & -\operatorname{Ra}_{c} \frac{\partial}{\partial x} \widehat{\mathcal{K}} \widehat{\mathcal{M}} & -\widetilde{\mathrm{Q}} \frac{\partial}{\partial z} \nabla^{2} \widehat{\mathcal{K}} \widehat{\mathcal{M}} & \sqrt{\operatorname{Ta}} \frac{\partial}{\partial z} \widehat{\mathcal{K}} \widehat{\mathcal{M}} \\
-\operatorname{Ra}_{c} \frac{\partial}{\partial x} \widehat{\mathcal{K}} \widehat{\mathcal{M}} & \operatorname{Ra}_{c} \frac{\partial}{\partial x} \widehat{\mathcal{K}} \widehat{\mathcal{N}} & 0 & 0 \\
-\widetilde{\mathrm{Q}} \frac{\partial}{\partial z} \nabla^{2} \widehat{\mathcal{K}} \widehat{\mathcal{M}} & 0 & \widetilde{\mathrm{Q}} \operatorname{Pr}^{-1} \nabla^{4} \widehat{\mathcal{K}} \widehat{\mathcal{M}} & 0 \\
\sqrt{\operatorname{Ta}} \frac{\partial}{\partial z} \widehat{\mathcal{K}} \widehat{\mathcal{M}} & 0 & 0 & -\sqrt{\operatorname{Ta}} \frac{\partial}{\partial z} \widehat{\mathcal{L}} \widehat{\mathcal{M}}
\end{array}\right) .
$$

Here the notations for new operators are inroduced

$$
\begin{gathered}
\widehat{\mathcal{L}}=q_{\alpha} R_{\alpha} \mathrm{Q} \frac{\partial^{4} \nabla^{2}}{\partial x^{2} \partial z^{2}}+\nabla^{2}\left(\nabla^{4}-\mathrm{Q} \frac{\partial^{2}}{\partial z^{2}}\right)\left(\nabla^{4}+q_{\alpha} R_{\alpha} \frac{\partial^{2}}{\partial x^{2}}\right), \\
\widehat{\mathcal{N}}=-\frac{\partial \nabla^{2}}{\partial z}\left(\nabla^{4}-\mathrm{Q} \frac{\partial^{2}}{\partial z^{2}}\right)\left(\nabla^{4}+q_{\alpha} R_{\alpha} \frac{\partial^{2}}{\partial x^{2}}\right)-q_{\alpha} R_{\alpha} \mathrm{Q} \frac{\partial^{5} \nabla^{2}}{\partial x^{2} \partial z^{3}} .
\end{gathered}
$$




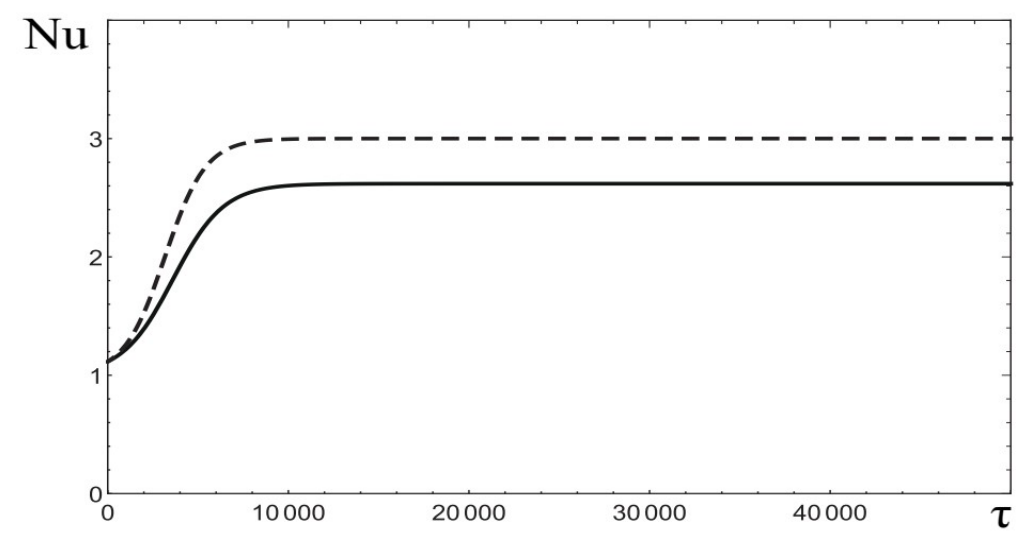

Fig. 8. Dependence of the Nusselt number $\mathrm{Nu}$ on time $\tau$. The dashed line shows the case without taking into account TM effects and the solid line with taking into account TM effects.

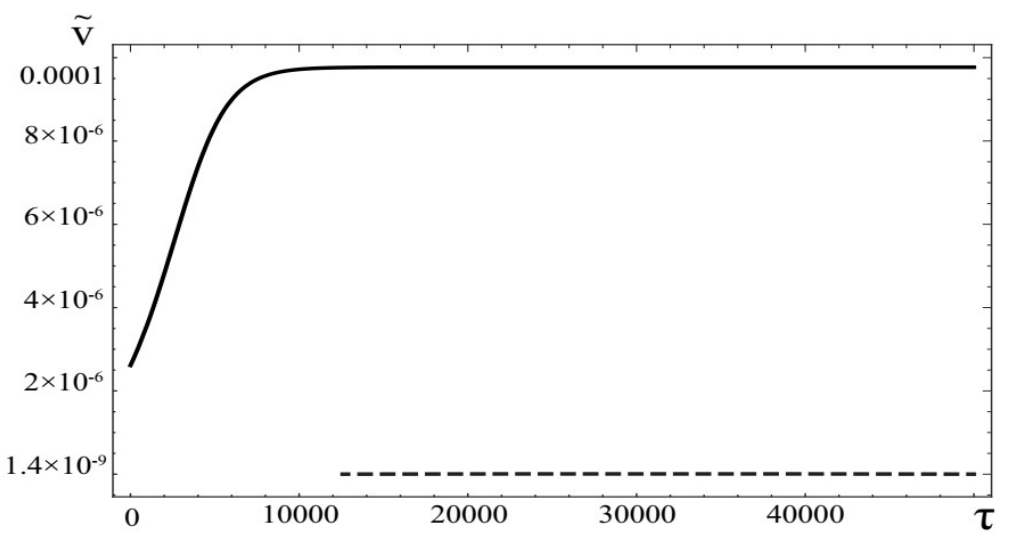

Fig. 9. Dependence of the generated magnetic field amplitude $\widetilde{v}(\tau)$ on time $\tau$. The steady-state amplitude $\widetilde{v}(\tau)$ taking into account TM effects (solid line) is approximately $10^{4}$ times greater than in the case without TM effects (dashed line).

By integrating (38), we can obtain a nonlinear equation for the amplitude $A(\tau)$, which refers to the GinzburgLandau equation or the Bernoulli differential equation with constant coefficients $\mathscr{A}_{1,2,3}$ :

$$
\mathscr{A}_{1} \frac{\partial A}{\partial \tau}-\mathscr{A}_{2} A+\mathscr{A}_{3} A^{3}=0 .
$$

Because of their cumbersome form, expressions $\mathscr{A}_{1,2,3}$ are given in Appendix B. In the limiting case, when TM effects are absent $\left(q_{\alpha}=0, R_{\alpha}=0\right)$, the equation (39) corresponds to the well-known result [32]. It is easy to obtain an analytical solution (39) with a known initial condition $A_{0}=A(0)$ :

$$
A(\tau)=\frac{A_{0}}{\sqrt{\frac{\mathscr{A}_{3}}{\mathscr{A}_{2}} A_{0}^{2}+\left(1-A_{0}^{2} \frac{\mathscr{A}_{3}}{\mathscr{A}_{2}}\right) \exp \left(-\frac{2 \tau \mathscr{A}_{2}}{\mathscr{A}_{1}}\right)}} .
$$

With the help of solution (40), we can determine the change in the magnitude of heat transfer (Nusselt number $\mathrm{Nu}$ ) and the amplitude of the generated magnetic field $\widetilde{v}(\tau)$ from time $\tau$ using formula (34). When performing calculations, we take the initial amplitude equal to $A_{0}=0.7$ and $\mathrm{Ra}_{2} \approx \mathrm{Ra}_{c}$, which corresponds to the smallness of the supercriticality parameter $\epsilon$. Constant convection parameters $\mathrm{Q}_{1}=\mathrm{Q} / \pi^{2}=17000$,
$\mathrm{T}_{1}=\mathrm{Ta} / \pi^{4}=10, \mathrm{R}_{1}=\mathrm{Ra} / \pi^{4}=20000, \mathrm{Pm}=1$, $\operatorname{Pr}=380000$ correspond to the previously adopted parameters of the Earth's core and the external magnetic field $B_{0}=10^{-1} \mathrm{~T}$. The profile of nonuniform rotation (Rossby number) is assumed to be Rayleigh, i.e. Ro $=-1$. The graph of the dependence of the Nusselt number $\mathrm{Nu}(\tau)$ for the above parameters is shown in Fig. 8 . Here, the dashed line corresponds to the case without taking into account TM effects, and the solid line - with taking into account TM effects. The graphs show the establishment of the final value $\mathrm{Nu}(\tau)$, due to the relationship between the number $\mathrm{Nu}(\tau)$ and the amplitude $A(\tau)$ (see formula $(34)$ ). The excess of the number $\mathrm{Nu}$ over one is due to the occurrence of convection. When TM effects are taken into account, heat transfer due to convection decreases, since convective instability reaches a stationary level at a lower value of the final amplitude. In this case, a part of the thermal energy is transformed into the energy of the generated magnetic field. The graph in Fig. 9 shows the establishment of a finite amplitude for the generated disturbances of the toroidal ( $Y$ component) magnetic field. Hence, it can be seen that when the TM effects are taken into account, the amplitude of the exciting magnetic field increased by about a factor of $10^{4}(!)$ 
Thus, convective processes taking into account TM phenomena play an essential role in the generation of magnetic fields in a nonuniformly rotating electrically conducting fluid.

\section{CONCLUSION}

In this paper, we consider the mechanism of magnetic field generation in a nonuniformly rotating electrically conductive fluid by TMI, which occurs at collinear temperature $\nabla T_{0}$ and thermoelectromotive force coefficient $\nabla \alpha$ gradients: $\left[\nabla \alpha \times \nabla T_{0}\right]=0$. The gradient of the thermoelectromotive force coefficient $\nabla \alpha$ is caused by the inhomogeneity of the chemical composition of the electrically conductive fluid. We have investigated the generation of a magnetic field by TMI in a nonuniformly rotating layer of an electrically conductive fluid in a constant vertical magnetic $\mathbf{B}_{0} \| O Z$ and a gravitational $-\mathbf{g} \| O Z$ field. In the linear approximation, we obtained the dispersion equation for axisymmetric perturbati- ons, from which the critical Rayleigh number $\mathrm{Ra}_{c}$ was determined for the stationary convection regime. The performed analysis of the stationary convection regime showed that the threshold for the development of convective instability decreases for a negative rotation profile $(\mathrm{Ro}<0)$. Also, the threshold for the development of convective instability taking into account TM effects also decreases for any profile of a nonuniform rotation, i.e. has a destabilizing effect. We investigated the weakly nonlinear stage of stationary convection taking into account TM effects using perturbation theory in the small supercriticality parameter $\epsilon=\sqrt{\left(\mathrm{Ra}-\mathrm{Ra}_{c}\right) / \mathrm{Ra}_{c}}$ of the stationary Rayleigh number $\mathrm{Ra}_{c}$ and obtained the nonlinear Ginzburg-Landau equation for the convection amplitude. From the solution of this equation, it follows that the generated toroidal magnetic field reaches a stationary level.

The results obtained in this work can find application in various problems of the magnetic geodynamo, as well as in laboratory studies on rotating magnetic convection taking into account thermomagnetic phenomena.
[1] J. Larmor, Rep. Brit. Assoc. Adv. Sci., 159 (1919).

[2] G. Moffat, Magnetic Field Generation in Electrically Conducting Fluids (Cambridge University Press, Cambridge, 1978).

[3] M. Steenbeck, F. Krause, K. H. Rädler, Z. Naturforsch. 21a, 369 (1966); https://doi.org/10.1515/zna-1966 $-0401$.

[4] Ya. Zeldovich, A. Ruzmaikin, D. Sokoloff, Magnetic Fields in Astrophysics (Gordon and Breach, New York, 1983).

[5] J. Parker, Conversations on Electric and Magnetic Fields in the Cosmos (Princeton University Press, Princeton, 2007).

[6] F. Krause, K. H. Rädler, Mean-field Magnetohydrodynamics and Dynamo Theory (Pergamon Press, Oxford, 1980).

[7] A. Ruzmaikin, A. Shukurov, D. Sokoloff, Magnetic Fields of Galaxies (Kluwer, Dordrecht, 1988)

[8] D. D. Sokoloff, R. A. Stepanov, P. G. Frick, Phys.-Usp. 57, 292 (2014); https://doi.org/10.3367/UFNe.0184. 201403 g. 0313.

[9] G. Rüdiger, R. Hollerbach, The Magnetic Universe. Geophysical and Astrophysical Dynamo Theory (WileyVCH Verlag GmbH, Weinheim, 2004).

[10] C. A. Jones, Dynamo Theory (University of Leeds, UK, 2007).

[11] A. Schlüter, L. Biermann, Z. Naturforsch. 5a, 237 (1950); https://doi.org/10.1515/zna-1950-0501.

[12] A. I. Laptukhov, Geomagn. Aeron. 20, 530 (1980).

[13] S. I. Braginsky, Geomagn. Aeron. 4, 698 (1964).

[14] A. Z. Dolginov, Sov. Phys. Usp. 30, 475 (1987); https: //doi.org/10.1070/PU1987v030n06ABEH002851.

[15] V. Urpin, Plasma Phys. Rep. 45(4), 366 (2019); https: //doi.org/10.1134/S1063780X19030103.

[16] P. H. Roberts, G. A. Glatzmaier, Geophys. Astrophys. Fluid Dyn. 94, 47 (2001); https://doi.org/10.1080/ 03091920108204131.

[17] E. Liverts, M. Mond, V. Urpin, Mon. Not. R. Aston. Soc. 404, 283 (2010); https://doi.org/10.1111/j.13
65-2966. 2010.16271.x

[18] Y. M. Shtemler, E. Liverts, M. Mond, Astron. Nachr. 333, 266 (2012); https://doi.org/10.1002/asna.201 211653.

[19] G. Montani, R. Benini, N. Carlevaro, A. Franco, Mon. Not. R. Aston. Soc. 436, 327 (2013); https ://doi .org/ $10.1093 / \mathrm{mnras} / \mathrm{stt} 1568$.

[20] L. E. Gurevich, B. L. Gel'mont, Sov. Astron. Astron. J. 11, 609 (1968).

[21] E. Velikhov, Sov. Phys. JETP 36, 995 (1959).

[22] S. A. Balbus, J. F. Hawley, Astrophys. J. 376, 214 (1991).

[23] M. I. Kopp, A. V. Tour, V. V. Yanovsky, J. Exp. Theor. Phys. 127, 1173 (2018); https://doi.org/10.1134/S1 $06377611812018 \mathrm{X}$.

[24] L. D. Landau, L. P. Pitaevskii, E. M. Lifshitz, Electrodynamics of Continuous Media (ButterworthHeinemann, 1984).

[25] V. N. Zharkov, Internal Structure of the Earth and Planets (Gordon and Breach, New York, 1983).

[26] S. Chandrasekhar, Hydrodynamics and Hydromagnetic Stability (Oxford University Press, London, 1961).

[27] O. N. Kirillov, F. Stefani, Y. Fukumoto, J. Fluid Mech. 760, 591 (2014); https://doi.org/10.1017/jfm.2014 .614 .

[28] P. Goldreich, D. Lynden-Bell, Mon. Not. R. Astron. Soc. 130, 125 (1965); https://doi.org/10.1093/mnras/13 0.2 .125 .

[29] E. Knobloch, K. Jullien, Phys. Fluids 17, 094106 (2005); https://doi.org/10.1063/1.2047592.

[30] W. V. R. Malkus, G. Veronis, J. Fluid Mech. 4(03), 225 (1958).

[31] R. Haberman, Elementary Applied Partial Differential Equations with Fourier Series and Boundary Value Problems (4th ed., Prentice-Hall, Inc., 2004).

[32] M. Kopp, A. Tur, V. Yanovsky, East Eur. J. Phys. 1, 4 (2019); https://doi.org/10.26565/2312-4334-2 020-1-01. 


\title{
ГЕНЕРАЦІЯ МАГНІТНОГО ПОЛЯ ТЕРМОМАГНІТНИМИ ЕФЕКТАМИ В ШАРІ ЕЛЕКТРОПРОВІДНОї РІДИНИ, ЯКИЙ НЕОДНОРІДНО ОБЕРТАЕТЬСЯ
}

\author{
М. Й. Копп ${ }^{1}$, К. М. Кулик ${ }^{1}$, А. В. Тур ${ }^{3}$, В. В. Яновський ${ }^{1,2}$ \\ ${ }^{1}$ Iнститут монокристалів, НАН Украӥни, просп. Науки, 60, 61001 Харків, Украӥна, \\ ${ }^{2}$ Харківсъкий національний університет імені В. Н. Каразіна, майдан Свободи, 4, 61022, Харків, Украӥна, \\ ${ }^{3}$ Тулузъкий університет [UPS], CNRS, Інститут вивчення астрофізики та планетологї̈, ав. Полковника Роше, \\ ВP 44346, 31028, Тулуза Cedex 4, Франціл
}

У статті розглянуто генерацію магнітних полів у шарі скінченної товщини електропровідної рідини, який неоднорідно обертається за рахунок термомагнітної (ТМ) нестійкості. Ця нестабільність виникає через градієнт температури $\nabla T_{0}$ і градієнт питомої термоерс $\nabla \alpha$. Необхідною умовою виникнення ТМ нестійкості $\epsilon$ колінеарність градієнтів температури $\nabla T_{0}$ і питомої термоерс $\nabla \alpha$. Розглянуто генерацію азимутального (тороїдального) магнітного поля в тонкому шарі електропровідної рідини, який неоднорідно обертається, що виникає через установлення позитивного зворотного зв'язку в системі завдяки ефектам неоднорідності питомої термоерс і Ледюка-Ригі. На вертикальних межах шару підтримуються постійні значення температури й питомої термоерс, причому нижня поверхня шару має вищу температуру, ніж верхня. У роботі досліджено лінійну й нелінійну стадії конвективної нестійкості з урахуванням генерації магнітного поля. Отримано загальне дисперсійне рівняння для осесиметричних $\partial / \partial \varphi=0$ збурень, що описує лінійну стадію конвективної нестійкості. У стаціонарному режимі конвекції встановлено, що генерація тороїдального магнітного поля за рахунок ТМ нестійкості істотно знижує поріг розвитку конвективної нестійкості в шарі замагніченої електропровідної рідини, який неоднорідно обертається за будь-якого профілю неоднорідного обертання (числа Россби Ro). Для опису нелінійних конвективних явищ використано локальну декартову систему координат, у якій неоднорідне обертання шару рідини зображено у вигляді обертання з постійною кутовою швидкістю $\Omega_{0}$ й азимутальним зсувом $\mathbf{U}_{0}(X)$, профіль швидкості якого локально лінійний. Унаслідок застосування методу теорії збурень для малого параметра надкритичності $\epsilon=\sqrt{\left(\mathrm{Ra}-\mathrm{Ra}_{c}\right) / \mathrm{Ra}_{c}}$ стаціонарного числа Релея $\mathrm{Ra}_{c}$ отримано нелінійне рівняння типу Гінзбурга-Ландау. Це рівняння описує еволюцію кінцевої амплітуди збурень. Числове розв'язання цього рівняння дозволило визначити теплоперенос у шарі рідини з ТМ-ефектами і без них. Показано, що амплітуда стаціонарного тороїдального магнітного поля помітно збільшується з урахуванням ТМ-ефектів.

Ключові слова: термоелектрорушійна сила, генерація магнітних полів, конвекція РелеяБенара, слабонелінійна теорія, рівняння Гінзбурга-Ландау. 